\title{
Subgroup deliberation and voting
}

\author{
Mark Thordal-Le Quement · Venuga Yokeeswaran
}

1 Abstract We consider three mechanisms for the aggregation of information in het-

M.T.-L. Quement (B) · V. Yokeeswaran

University of Bonn, Bonn, Germany

e-mail: mark.le.quement@uni-bonn.de 
We examine two intuitive communication protocols in heterogeneous committees that vote under Unanimity: Plenary Deliberation and Subgroup Deliberation. Our aim is to rank these communication protocols w.r.t. simple Private Voting as well as among each other. We proceed in two main steps, by first isolating a set of equilibrium predictions for each protocol and then comparing these predictions as a means of comparing protocols.

The first step of our analysis is as follows. For each communication protocol as well as for Private voting, we restrict ourselves to a class of simple equilibria and call these respectively Simple Subgroup Deliberation equilibria, Simple Plenary Deliberation equilibria and Simple No Deliberation Equilibria. The restrictions on strategies embedded in the term simple are mild in the case of Private Voting and in contrast significant in the case of Subgroup and Plenary Deliberation. Within the classes of equilibria considered, we furthermore only consider so called reactive equilibria, i.e. equilibria in which the same decision is not always made.

The second step of our analysis unfolds as follows. Having isolated a (non empty) set of equilibrium predictions for each of our protocols, we ask two specific questions. First, do there always exist reactive Simple Subgroup Deliberation and reactive Simple Plenary Deliberation equilibria that are Pareto improving w.r.t. any reactive Simple No Deliberation equilibrium? Secondly, does there always exist some reactive Simple Subgroup Deliberation equilibrium that is Pareto improving w.r.t any reactive Simple Plenary Deliberation equilibrium? Our answer to both questions is positive. The first result reveals that the two communication protocols dominate No Deliberation in a robust sense, given the mild restrictions imposed on strategies under Private Voting. Our second result shows that Subgroup Deliberation dominates Plenary Deliberation if one is willing to accept the significant restrictions that we impose on strategies under Plenary Deliberation. The latter form of dominance is thus admittedly significantly less general than the first form of dominance established. Modulo this important caveat, we thus obtain a complete ranking of the three voting mechanisms considered: Subgroup Deliberation dominates Plenary Deliberation which itself dominates Private Voting.

Among the plethora of potential communication protocols, we choose to focus on Plenary Deliberation and Subgroup Deliberation because we deem them intuitive and empirically relevant for the very reason that they are uncomplicated. The Plenary Deliberation protocol is equivalent to the common practice of straw votes: Each committee member simultaneously sends a public message chosen from a binary message space. Subgroup Deliberation restricts deliberation to homogeneous Subgroups. Examples of the latter protocol abound. In parliaments or parliamentary committees, party fellows often separately consult and reach a common stance before voting. Prior to faculty meetings, professors with related research agendas may meet separately. The key distinction between Plenary and Subgroup Deliberation resides in the a priori restriction that they place on information pooling. While Plenary Deliberation theoretically allows for a larger amount of information pooling than Subgroup Deliberation, our result is that Subgroup Deliberation however generates superior information sharing in equilibrium than Plenary Deliberation, when committees are heterogeneous. In other words, our finding is that Subgroup Deliberation a posteriori generates more efficient information sharing than Plenary Deliberation for the very reason that it $a$ priori restricts information sharing. 
Early contributions in the literature on collective decision making and information aggregation focus on Private Voting and compare different voting rules. Seminal contributions such as Feddersen and Pesendorfer (1998), Gerardi (2000) and Duggan and Martinelli (2001) negatively single out Unanimity. Meirowitz (2002) adds a caveat

to the above. The author examines a model featuring a continuum signal space as well as (at least nearly) perfectly informative signals and finds that full information equivalence obtains in the limit also for Unanimity.

Newer contributions add a stage of cheap talk communication prior to the vote. Gerardi and Yariv (2007) find that if one makes imposes no restriction on the communication protocol used, all non unanimous voting rules are equivalent in the sense that they induce the same set of equilibrium outcomes. Gerardi and Yariv (2007) contrasts with most of the remaining literature on cheap talk deliberation, which has instead examined specific protocols as well as simple equilibria. Most contributions have focused on the simultaneous Plenary Deliberation protocol and the truthful deliberation/sincere voting equilibrium (TS equilibrium). Coughlan (2000) shows that if preferences are known and substantially heterogeneous, the TS equilibrium does not exist. Austen-Smith and Feddersen (2006) show, within a generalized version of the classical Condorcet jury model, that uncertainty about preferences can render the TS equilibrium compatible with substantial heterogeneity, provided that the voting rule is not Unanimity. Meirowitz (2007), Van Weelden (2008) and Le Quement (2012) add further caveats to the analysis of Austen-Smith and Feddersen (2006). Finally, Deimen et al. (2014) show that if one considers a richer information structure featuring conditionally correlated signals, the TS equilibrium is compatible with a positive probability of ex post disagreement.

The question of the welfare properties of different protocols and equilibria has by and large been eluded. Clearly, in a homogeneous committee, the TS equilibrum implements the welfare maximizing decision rule, but little is known beyond this insight. Doraszelski et al. (2006) study a two persons setting with heterogeneous players who communicate simultaneously before voting under Unanimity. In equilibrium, information transmission is noisy, but communication is advantageous. Hummel (2010) identifies conditions under which Subgroup Deliberation ensures no errors in asymptotically large and homogeneous committees. Wolinsky (2002) analyzes an expert game and shows that a Principal can sometimes gain by strategically grouping experts into optimally sized Subgroups that pool information before reporting to him.

This paper complements existing literature on four aspects. First, it examines a little studied communication protocol, Subgroup Deliberation, that constitutes an alternative to Plenary Deliberation in heterogeneous committees in which types are publicly known. Second, it proposes a simple equilibrium scenario under Plenary Deliberation, for heterogeneous committees in which the TS equilibrium does not exist (so called minimally diverse committees; see Coughlan 2000). Third, it provides a first attempt at a general clarification of the relative (Pareto) welfare properties of Private Voting, Subgroup and Plenary Deliberation. Finally, from a technical perspective, it introduces a simple method for the Pareto comparison of equilibria arising under different proto- 
cols in heterogeneous committees, which simply invokes a hypothetical sequence of best responses by different juror types.

The paper is organized as follows. Section 2 introduces the basic jury model as well as the different communication protocols and equilibria that we consider. Section 3 provides a positive analysis of the equilibrium sets corresponding to the respective protocols under the imposed restrictions on strategy profiles. Section 4 compares the identified equilibria in terms of their Pareto welfare properties and thereby provides a tentative ranking of protocols. Section 5 concludes. Proofs are mostly relegated to Appendixes 1,2 and 3.

\section{The Model}

\subsection{Setup}

Suppose a jury composed of $n$ members. A defendant is being judged and is either guilty $(G)$ or innocent $(I)$ with equal prior probability. The jury must decide whether to convict $(C)$ or acquit $(A)$ him. Each juror casts a vote in favour of either conviction or acquittal. The voting rule is Unanimity: The defendant is convicted if and only if all jurors vote for conviction.

Each juror receives a single private signal prior to the vote. A signal $s \in\{i, g\}$ indicates either guilt or innocence. A signal is "correct" with probability $p \epsilon_{2}{ }^{*}, 1$, i.e. $P(s=g \mid G)=P(s=i \mid I)=p$, while $P(s=i \mid G)=P(s=g \mid I)=1-p$. Juror signals are i.i.d. Let $|g|$ denote the total number of $g$-signals received by the jury. The conditional probability $P(G|| g \mid=k)$ that the defendant is guilty given $|g|=k$ in an $n$ persons jury is given as follows:

$\beta(p, k, n):=\frac{B(p, k, n)}{B(p, k, n)+B(1-p, k, n)}$, where $B(p, k, n):=\stackrel{-}{n}_{k}^{-} p^{k}(1-p)^{n-k}$.

For $j \in\{1, \ldots, n\}$, each jury member $j$ 's preferences, are determined by a commonly known parameter $q^{j} \in(0,1)$. A juror's payoff function is given as follows: Define $U_{j}(C \mid I)=-q^{j}$ as the utility obtained by juror $j$ when the defendant is convicted despite being innocent, and $U_{j}(A \mid G)=-\left(1-q^{j}\right)$ as the utility obtained when the defendant is acquitted but guilty. The utility related to remaining combinations of state and action (acquittal of an innocent or conviction of a guilty) is normalized to 42 . Suppose a mechanism $M$ yielding a probability $P(C \mid I)$ of convicting an innocent defendant and a probability $P(A \mid G)$ of acquitting a guilty defendant. The expected utility of juror $j$ under mechanism $M$ is given as follows:

$$
U_{j}(M):=-q^{j} P(C \mid I) P(I)-\left(1-q^{j}\right) P(A \mid G) P(G) .
$$

Given this utility function, a juror $j$ prefers conviction to acquittal whenever his posterior probability that the defendant is guilty exceeds $q^{j}$. The parameter $q^{j}$ thus 
measures the juror's degree of aversion to wrongful conviction. The higher $q^{j}$, the more evidence of guilt is required for juror $j$ to prefer conviction.

Juror preferences are heterogeneous and fall into two homogeneous categories. The jury contains $n_{D}$ doves $(D)$ with preferences $q_{D}$ and $n_{H}$ hawks $(H)$ with preferences $q_{H}$, where $q_{H}<q_{D}$ and $n_{D}+n_{H}=n$. We assume that at least one of the two preference types is present at least twice in the committee. We refer to the allocation of committee seats among preference types as the jury composition. For each $j \in\{H, D\}$,

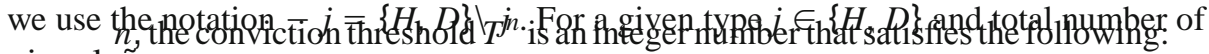
signals

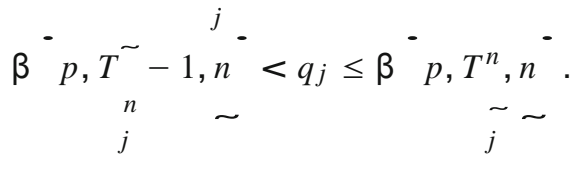

We make the following assumptions about preferences. First,

$$
\text { A.1: } T_{D}^{n}-T_{H}^{n}:=m \geq 2 .
$$

In other words, in a putative equilibrium in which all $n$ signals would be publicly revealed before the vote, at least two signal profiles would cause disagreement between the different juror types. The restriction is mild. Assuming $m=1$ typically imposes closely aligned preferences within the context of reasonably large committees in which many private signals are available. Second,

$$
\text { A.2: } T^{n_{j}} \in{ }^{-} 1, \ldots, n, \forall j \in\{H, D\} \text {. }
$$

This means that if jurors of a given preference type $j$ were to decide optimally on the basis of their $n_{j}$ signals, they would sometimes acquit and sometimes convict. Finally,

(1)

$$
\text { A.3: } q_{D}>\frac{1}{2}
$$

This implies that a dove favours conviction only if the probability that the defendant is guilty exceeds $\frac{1}{2}$. This requirement matches the jury setting, where the "voir dire" selection process eliminates jurors that are excessively prone to convict. The assumption is used in proving our welfare results and we do not claim that it is necessary.

Throughout this paper, we examine games exhibiting the following timing. In stage 0 , jurors receive private signals. In stage 1 , jurors communicate according to an exogenously fixed communication protocol. In stage 2 , jurors simultaneously cast a vote. In stage 3 , the defendant is convicted if and only if $n$ conviction votes were cast.

\subsection{Communication protocols and equilibria}

We now introduce the three communication protocols that are the object of our analysis. No Deliberation (ND) simply specifies that no message is sent. Plenary Deliberation (PD) specifies that each juror simultaneously sends a message $m \in\{i, g\}$ that is 
observed by all jurors. Subgroup Deliberation (SD) specifies that each juror simultaneously sends a message $m \in\{i, g\}$ that is observed only by jurors of his preference type.

Protocols are orderable according to the physical restraints that they impose on communication. The first, No Deliberation, fully prohibits information sharing among jurors. The second, Plenary Deliberation, potentially allows for full pooling of information among all jurors. The third, Subgroup Deliberation, prohibits communication between jurors of different preference types and only allows information pooling to take place within Subgroups of homogeneous jurors. Note that under Plenary as well as Subgroup Deliberation, we assume that communication is simultaneous, i.e. can be interpreted as simple straw votes preceding the actual vote. This is restrictive and must be distinguished from the free form communication considered in Gerardi and Yariv (2007).

Weintroduce a set of general definitions and restrictions on strategy profiles. A symmetric strategy profile specifies that jurors of the same preference type follow the same strategy. Monotonous strategies are s.t. information sets providing higher evidence of guilt are associated with a higher probability of voting for conviction. Throughout the analysis, we restrict ourselves to symmetric and monotonous strategies, in line with previous work on information aggregation and voting. We furthermore apply the follow heuristic principle. For a given protocol, we ignore the possibility of mixing (in communication as well as in voting) as long as such a restriction does not leave us only with trivial equilibria in which the same decision (either $C$ or $A$ ) is always made. This is true of the PD and the SD cases. It is in contrast not true under ND and we thus consider the possibility of mixed voting under the latter prococol. We now present in detail the strategy profiles and equilibria that our analysis focuses on. Our focus is on perfect bayesian equilibria, which we simply call equilibria in what follows.

\subsection{No deliberation}

Under ND, jurors condition their votes exclusively on their own signal. We use the term no deliberation strategy instead of the standard term private voting strategy to describe the voting behavior of jurors under this protocol. A symmetric no deliberation strategy profile is characterized by a vector of mixing probabilities $\underset{i}{\sigma^{H}}, \sigma_{g}^{H}, \sigma_{i}^{D}, \sigma_{g}^{D}$, where $\sigma_{s}^{j}$ denotes the probability that a single juror of type $j$ votes for conviction given a signal $s \in\{i, g\}$. Let $p i \mathrm{v}_{j}$ denote the event in which a given juror of preference type $j$ is pivotal in the sense that the final decision changes with the juror's vote. Let $\mathrm{Y}_{G}^{j}$ and $v^{j}$ denote the likelihood that a juror of preference type $j$ votes for conviction given respectively state $G$ or $I$. We have

$$
\begin{aligned}
\mathrm{Y}_{G}^{j} & =p \sigma_{g}^{j}+(1-p) \sigma_{j}^{j}, \\
\mathrm{Y}_{j}^{j} & =(1-p) \sigma_{g}+p \sigma_{i} .
\end{aligned}
$$

Define furthermore the indicator function $Y(j, k)$ as follows. For $j, k \in\{H, D\}$, $Y(j, k)=1$ if $j=k$ while $Y(j, k)=0$ otherwise. Clearly, given the Unanimity rule, 
$P\left(G \mid s, p i \mathrm{v}_{j}\right)$

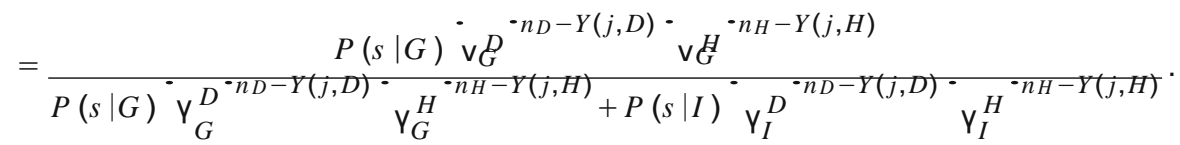

We call symmetric and monotonous no deliberation strategy profiles simple $N D$ profiles (SND). If an SND profile is s.t. the defendant has a positive ex ante chance of both being acquitted or convicted, we call it a reactive SND profile. If an SND profile is s.t. the defendant is either always acquitted or always convicted, we call it a non reactive SND profile.

Lemma1 Under the ND protocol, a reactive SND profile $\sigma_{i}^{H},{ }_{g}^{H}, \sigma_{i}^{D}, \sigma_{g}^{D}$ constitutes an equilibrium iff, $\forall j \in\{H, D\}, \forall s \in\{i, g\}$ :

$$
\begin{aligned}
& P\left(G \mid s, \text { piv } \mathrm{v}_{j}\right)=q_{j} \text {, when } \sigma_{s}^{j} \in(0,1), \\
& P\left(G \mid s, \text { piv } \mathrm{v}_{j}\right) \leq q_{j}, \text { when } \sigma_{s}^{j}=0, \\
& P\left(G \mid s, \text { piv }_{j}\right) \geq q_{j}, \text { when } \sigma_{s}^{j}=1 .
\end{aligned}
$$

Proof The above conditions are standard (see for example Feddersen and Pesendorfer 1998) and their proof is therefore omitted.

Under the ND protocol, a reactive SND profile that constitutes an equilibrium is called a reactive SNDE.

\subsection{Plenary deliberation}

Under the PD protocol, consider first the strategy profile in which all jurors first truthfully reveal their signals while there is a threshold $t \in\{1, \ldots, n\}$ s.t. all jurors vote for conviction iff at least $t g$-signals have been announced. We know from Coughlan (2000) that no such strategy profile constitutes an equilibrium of the game if $m \geq 1$.

We instead examine a strategy profile that is given as follows. In Stage 1, jurors of type $j$ truthfully reveal their signal while jurors of type $-j$ simply always sends the message $g$ and thus babble. In Stage 2, the voting decision of both juror types is conditioned on the number of $g$-signals announced by type $j$. That is, there is a $t_{j} \in{ }^{-} 0,1, \ldots, n_{j}, n_{j}+1^{-}$such that: (1) all jurors vote for conviction if at least $t_{j}$ $g$-signals have been announced by jurors of type $j$ and (2) all jurors vote for acquittal otherwise. We call this strategy profile a simple PD strategy profile (SPD), thereby emphasizing the fact that one could envisage more complex strategy profiles under the PD protocol, for example involving noisy communication or mixed voting. We furthermore call an SPD profile a reactive SPD profile if $t_{j} \in{ }^{-} 1, \ldots, n_{j}{ }^{-}$, i.e. if jurors have a positive ex ante chance of unilaterally voting for both acquittal and conviction. If an SPD strategy profile is s.t. the defendant is either always acquitted or always convicted, we call it a non reactive SPD strategy profile.

Our restriction to pure strategies leaves us exclusively with equilibria in which doves truthtell while hawks babble. Truthtelling by doves appears natural given the 
allocation of power across types, which unambiguously favours doves. Given a profile of public information, if doves favour conviction, then hawks do so as well and will thus not veto such an outcome. If doves instead favour acquittal, they can furthermore always veto a conviction. In principle, doves can thus always get their way. The fact that hawks babble in the equilibria that we examine also appears quite natural in the light of this power allocation. As a matter of fact, we conjecture that there generally exists no symmetric and monotonic equilibrium in which an individual hawk is with positive probability pivotal at the communication stage. The argument behind this would be as follows. Given the preference misalignment assumed between doves and hawks $(m>1)$, conditional on the event of being pivotal at the communication stage, a hawk favours conviction independently of his own signal. Consequently, if assumed to communicate informatively, a hawk will always favour announcing a $g$-signal.

Lemma 2 Under the PD protocol, a reactive SPD profile characterized by $t_{j} \in$ $\cdot 1, \ldots, n_{j}{ }^{-}$constitutes an equilibrium iff:

$$
\beta^{\circ} p, t_{j}-1, n_{j}{ }^{\circ}<q_{j} \leq \beta^{\circ} p, t_{j}, n_{j} \cdot
$$

and

$$
q_{-j} \leq \beta^{\circ} p, t_{-j}, n_{-j}+1^{\cdot} .
$$

Proof The double inequality (7) is necessary and sufficient for a juror of type $H$ not to have a strict incentive to deviate either at the communication or at the voting stage. The inequality (8) is necessary and sufficient to ensure that preference type $-j$ is always willing to vote for conviction whenever at least $t_{j}$ guilty signals are announced by jurors of type $j$.

Under the PD protocol, a reactive SPD profile that constitutes an equilibrium is called a reactive SPDE. One may be uneasy with our ignoring the possibility of mixing at the voting stage. Our justification is purely practical: Including equilibria featuring mixed voting following truthtelling would be a daunting task for reasons that we explain in what follows. Recall that type $j$ is the type that is truthelling in the communication stage and consider an equilibrium featuring truthtelling followed by possibly mixed voting. Let $A_{-}^{i}, \theta_{-j}^{g}$ describe the (possibly mixed) voting strategy of type $-j$, where $\theta_{-j}^{s}$ is the probability of voting $C$ given signal $s \in\{i, g\}$. Symmetric mixed voting by jurors of type $j$ requires indifference between decisions $A$ and $C$ at a given information set. This implies that given a voting strategy $\mathrm{A}_{j}^{i}, \theta_{-j}^{g}$ of type $-j$, the mixed voting strategy of type $j$ must be summarized by a vector $\left(t_{j}, \theta_{j}\right)$ specifying the following voting behavior. When Subgroup $j$ holds $t_{j} g$-signals, each of its members votes $C$ with probability $\theta_{j}$. When Subgroup $j$ holds strictly more (less) than $t_{j} g$-signals, all $j$-types convict (acquit). Furthermore, the conditional probability of guilt, conditional on $t_{j} g$-signals in Subgroup $j$ and on the assumption that all jurors of type $-j$ convict, is equal to $q j$. In order to characterize the set of equilibria featuring truthtelling followed by possibly mixed voting, one would thus have to identify an equilibrium vector given by $\left(t_{j}, \theta_{j}, \theta_{-j}^{i}, \theta_{-j}^{g}\right)$. This task is substantially more complicated 
than identifying a unique threshold $t_{j}$ (equivalent to $\left.\left(t_{j}, 1,1,1\right)\right)$ as we do. Furthermore, the increased complexity would carry over to the subsequent welfare exercise.

\subsection{Subgroup deliberation}

Under the SD protocol, we consider strategy profiles that are entirely characterized by a vector of thresholds $t=\left(t_{H}, t_{D}\right)$. In Stage 1, jurors simultaneously truthfully disclose their private signal to members of their Subgroup by sending a message identical to their signal. In Stage 2, all members of Subgroup $j$ vote for conviction if the total number of guilty messages received among members of Subgroup $j$ is weakly larger than $t_{j}$, and otherwise all vote for acquittal. We call this strategy profile a simple $S D$ profile (SSD), thereby emphasizing the fact that one could construct more complex profiles under the SD protocol, for example involving noisy communication or mixing at the voting stage. We focus on SSD profiles that are such that the defendant has a positive ex ante chance of both being acquitted or convicted. We call such SSD profiles reactive SSD profiles and these come in two subforms. A type 2 reactive SSD profile is

a SSD profile in which $t_{j} \in{ }^{-} 1, \ldots, n_{j}{ }^{-}$for each $j \in\{H, D\}$. A type 1 reactive $S S D$ profile is a reactive SSD profile in which one Subgroup $j \in\{H, D\}$ adopts $t_{j}=0$, while Subgroup $-j$ adopts a threshold $t_{-j} \in{ }^{-} 1, \ldots, n_{-j}{ }^{-}$. If an SSD strategy profile is s.t. the defendant is either always acquitted or always convicted, we call it a non reactive SSD strategy profile.

We comment on key restrictions here. Given perfectly identical Subgroup preferences, focusing on outcomes featuring truthtelling appears natural. In contrast, one may be uneasy with our ignoring the possibility of mixing at the voting stage. Our justification is, as in the case of PD, purely practical: Including equilibria featuring mixed voting following truthtelling would be a daunting task. Symmetric mixed voting by jurors of type $j$ requires indifference between decisions $A$ and $C$ at a given information set. This implies that given a strategy of type $-j$ featuring truthtelling followed by (possibly mixed) voting, the mixed voting strategy of type $j$ is summarized by a vector $\left(t_{j}, \theta_{j}\right)$, as in the case of mixed voting under PD described above. In order to characterize the set of equilibria featuring truthtelling followed by possibly mixed voting, one would thus have to identify an equilibrium vector given by $\left(t_{H}, \theta_{H}, t_{D}, \theta_{D}\right)$. This task is substantially more complicated than identifying a pair $\left(t_{H}, t_{D}\right)$ (equivalent to $\left.\left(t_{H}, 1, t_{D}, 1\right)\right)$ as we do. Furthermore, the increased complexity would carry over to the subsequent welfare exercise. More equilibria means more equilibria to compare, and mixed voting equilibria might not easily compare with each other or with pure voting equilibria. A final justification is the presumably limited impact of mixed voting on the set of implementable decision rules. When a Subgroup $j$ is not excessively small, truthtelling in Subgroups implies a large array of revealed Subgroup signal profiles, out of which no more than one could induce randomized voting, as explained. When Subgroups are large, randomization in voting by a given preference type will thus only occur rarely in any given equilibrium and is thus arguably unlikely to heavily affect the type of implementable decision rules.

We now characterize conditions under which a given reactive SSD profile constitutes an equilibrium. Let $|g|_{j}$ stand for the number of guilty signals held by Subgroup 
$j$. Let ${ }^{\bullet}|g|_{j}=t_{j},|g|_{-j} \geq t_{-j}{ }^{\bullet}$ denote the event in which Subgroup $j$ holds exactly $t_{j} g$-signals while Subgroup $-j$ holds at least $t_{-j} g$-signals.

Lemma 3 a) Under the $S D$ protocol, a type 2 reactive $S S D$ profile given by $\left(t_{H}, t_{D}\right)$, where $t_{j} \in{ }^{-} 1, \ldots, n_{j}{ }^{-} \forall j \in\{H, D\}$, constitutes an equilibrium iff:

$$
P^{\circ} G^{\circ}|g|_{j}=t_{j}-1,|g|_{-j} \geq t_{-j}{ }^{\circ}<q_{j} \leq P^{\circ} G^{\circ}|g|_{j}=t_{j},|g|_{-j} \geq t_{-j} \cdot .
$$

b) Under the $S D$ protocol, a type 1 reactive $S S D$ profile given by $\left(t_{H}, t_{D}\right)$, where for some $j \in\{H, D\}, t_{j} \in{ }^{-} 1, \ldots, n_{j}{ }^{-}$and $t_{-j}=0$, constitutes an equilibrium iff (9) is true and

$$
q_{-j} \leq P^{*} G^{\circ}|g|_{-j}=0,|g|_{j} \geq t_{j} \cdot .
$$

\section{Proof See in Appendix 1.}

Under the SD protocol, a type 1 or type 2 reactive SSD profile that constitutes an equilibrium is called respectively a type 1 or type 2 reactive SSDE.

The idea behind reactive SSDEs is that each homogeneous Subgroup $j$ votes as one person endowed with $n_{j}$ signals. The SD protocol defines a sequential game in

which individuals first communicate in Subgroups and then vote. We start with a discussion of Point (a). The key insight is that condition (9) simultaneously ensures no strict deviation incentives both at the communication and at the voting stage. As

to Point (b), which characterizes type 1 reactive SSDEs, note that the behavior of Subgroup $j$, as specified in (9), is the same as if it were deciding alone and voting ex post optimally after fully pooling its information. Assuming that Subgroup $-j$ convicts indeed provides no indication regarding the signal profile of the latter, as it always convicts. Subgroup $-j$, on the other hand, simply always convicts under the assumption that Subgroup $j$ is convicting.

Our analysis unfolds in two steps. Section 3 provides a descriptive analysis of reactive SND, SPD and SSD equilibria. Section 4 analyzes the comparative welfare properties of reactive SSDEs, SPDEs and SNDEs.

\section{Positive analysis}

Lemma 4 Under the ND protocol, a unique reactive SND profile constitutes an equilibrium. It is given by $(\sigma \stackrel{g}{=}=1, \sigma H=1, \sigma \underset{g}{=}=1, \sigma D=y)$, where $y \in(0,1)$ if $T^{n_{D}}$ $T_{D}^{n_{D}}<n_{D}$ and $y=0$ if $T_{D}^{n_{D}}=n_{D}$.

Proof See in Appendix 2.

The unique reactive SNDE, under our restrictions, is thus one in which hawks always convict, while doves vote as if they were an independent committee voting privately under Unanimity. The voting behavior of doves replicates the equilibrium characterized in Feddersen and Pesendorfer (1998). The key property of the unique reactive SNDE is that only the information of doves is aggregated, and typically 
imperfectly so, due to the fact that voting is private. As a final comment, note that our assumption that $m>1$ is key to eliminating a large amount of potential equilibrium scenarios under ND. When the doves are sufficiently biased towards acquittal (in relative terms), the assumption that all doves convict provides strong indication of guilt and unambiguously outweighs an individual hawk's information.

Lemma 5 Under the PD protocol, a unique reactive SPD profile constitutes an equilibrium. It is characterized by $t_{D}=T_{D}^{n_{D}}$.

Proof See in Appendix 2.

As already mentioned, it is intuitive that there exists an equilibrium in which doves publicly reveal their information, given that Unanimity voting effectively delegates decision power to them. This effective decision power of doves similarly explains why there is no reactive Simple Plenary Deliberation equilibrium in which hawks truthfully reveal their information. While the common feature of the unique reactive SNDE and SPDE is that hawks effectively delegate decision making to the doves, the difference between the two equilibria resides in the way doves aggregate their information. In the unique reactive SNDE, doves do not pool their information and thus always aggregate their information imperfectly if $T_{D}^{n_{D}}<n_{D}$. In the unique reactive SPDE, doves always fully pool their information, coordinate votes and aggregate their information optimally.

\section{Lemma 6 Under the $S D$ protocol:}

(a) At least one reactive SSD profile constitutes an equilibrium.

(b) If there exist $K>1$ reactive SSDEs, then there exists a vector $t_{H}^{1}, t_{D}^{1}{ }_{D}$ s.t. the set of SSDEs is given by:

$$
{ }_{H}^{-t^{1}}, t_{D} \quad, t_{H}-1, t_{D}^{1}+1^{-}, \ldots, \quad t_{H}^{1}-K+1, t_{D}^{1}+K-1 .
$$

Proof See in Appendix 2.

Here again, there always exists an equilibrium satisfying our restrictions on strategies. In contrast to the sets of reactive SNDEs and reactive SPDEs, the set of reactive SSDEs may however contain more than one element. Point b) shows that if there exist several reactive SSDEs, these are orderable in terms of their degree of polarization. Among two reactive SSDEs, we say that the equilibrium with lower $t_{H}$ and higher $t_{D}$ is more polarized, because each of the Subgroups acts more in accordance with its own relative bias.

This concludes our descriptive equilibrium analysis, given our restrictions on strategy profiles. Having identified a set of equilibrium scenarios for each protocol, we may now proceed to a welfare comparison of the identified equilibria, aimed at producing a tentative ranking of the three considered protocols.

\section{Normative analysis}

We say of an equilibrium that it is strongly Pareto dominant w.r.t. another equilibrium if both preference types obtain a strictly higher expected welfare in the first equilibrium. 
This subsection proceeds in three parts. First, Proposition 1 provides a Pareto welfare comparison of the unique reactive SPDE to the unique reactive SNDE. It establishes

that the first equilibrium either strongly Pareto dominates the latter or is outcome equivalent to it. Second, Proposition 2 shows that when the set of reactive SSDEs is not a singleton, its elements are ordered in the strong Pareto sense. Third, Proposition

3 Pareto compares reactive SSDEs to the unique reactive SPDE. When the set of reactive SSDEs is not a singleton, the Pareto dominated equilibrium within this set either strongly Pareto dominates the unique reactive SPDE or is outcome equivalent to it. When the set of reactive SSDEs is a singleton, its unique element either strongly Pareto dominates the unique reactive SPDE or is outcome equivalent to it.

We add a comment on the interpretation of our theoretical exercise. Our reference to a jury setting may appear problematic because jury deliberations typically do not allow for Subgroup Deliberation. We see our analysis as a contribution to a normative debate aiming at potentially redesigning existing deliberation protocols in juries. In this perspective, considering new designs that are not in use seems legitimate. To the extent that one endorses our (admittedly restrictive) predictions for the different protocols, our welfare results would imply that members of a heterogeneous jury would unanimously agree to deliberate separately, if given the choice between Plenary Deliberation and Subgroup Deliberation.

First, Jurors' ethnic or social background does appear to be a partial predictor of their preferences. Furthermore, the ethnic or social background of a person is at least imperfectly inferable from observable attributes (physical, verbal, psychological, etc).

\section{Proposition 7 Reactive SPDE vs reactive SNDE.}

(a) If $T_{D}^{n_{D}}=n_{D}$, the unique reactive SPDE is outcome equivalent to the unique reactive $S N D E$.

(b) If $T_{D}^{n_{D}}<n_{D}$, the unique reactive SPDE is strongly Pareto dominant w.r.t the unique reactive $N S D E$.

\section{Proof See in Appendix 3.}

As already mentioned, the unique reactive SNDE allows to optimally aggregate the information held by doves only if $T_{D}^{n}=n_{D}$, while the unique reactive SPDE always allows to achieve an optimal aggregation of the doves' information. This fact is reflected in the distinction between cases a) and b).

Our assumption that $q_{D}>{ }^{1}$ is key to showing that the unique reactive SPDE strongly Pareto dominates the unique reactive SNDE if $T_{D}^{n_{D}}<n_{D}$. If $q_{D}>\frac{1}{2}$, a key aspect is that, maintaining the assumption of a unilateral conviction vote by hawks, transiting from private voting by doves (call this the private scenario) to an optimal aggregation of pooled signals by doves (call this the pooled scenario) leads to an increase in the ex ante probability of conviction and is thereby strictly beneficial to hawks. In the unique reactive SNDE, hawks indeed suffer from the doves' lack of willingness to convict. An adjustment in the doves' behavior that mitigates this reluctance without dramatically overshooting is thus naturally advantageous for hawks.

We now expand on the reason behind the fact that our condition requires a high enough $q_{D}$. As $q_{D}$ increases, the probability of a unilateral conviction vote admittedly 
decreases under both scenarios (private and pooled) considered above, but the key aspect is that this probability decreases faster under the first than under the second scenario. In the private scenario, a unilateral conviction vote by doves requires that every dove either receives a $g$-signal or, conditional on receiving an $i$-signal, votes for conviction, the latter event happening with probability $y\left(p, q_{D}, n_{D}\right) \in(0,1)$. For very high values of $q_{D}, y\left(p, q_{D}, n_{D}\right)$ is however very low and furthermore tends to 0 very fast as $q_{D}$ tends to $\beta\left(p, n_{D}-1, n_{D}\right)$. In contrast, as $q_{D}$ increases and tends to $\beta\left(p, n_{D}-1, n_{D}\right)$, the likelihood of a coordinated conviction vote by doves in the pooling scenario decreases slowly and without tending to 0 . It is therefore quite intuitive that for $q_{D}$ large enough, transiting from the private to the pooling scenario increases the likelihood of a unilateral conviction vote by doves.

Before going on to the final step of our normative analysis, which provides a comparison of reactive SSDEs to the unique reactive SPDE, we establish the preliminary result that the set of reactive SSDEs is fully orderable in the Pareto sense.

\section{Proposition 8 Reactive SSDEs.}

If $\left(t_{H}, t_{D}\right),\left(t_{H}-1, t_{D}+1\right)$ are two reactive SSDEs, then $\left(t_{H}, t_{D}\right)$ is strongly Pareto improving w.r.t. $\left(t_{H}-1, t_{D}+1\right)$.

Proof Consider two reactive SSDEs $\left(t_{H}-1, t_{D}+1\right)$ and $\left(t_{H}, t_{D}\right)$. First, as proved in Appendix 3, transiting from $\left(t_{H}-1, t_{D}+1\right)$ to $\left(t_{H}-1, t_{D}\right)$ is beneficial for the preference type $H$ given our assumption that $m>1$. Second, transiting from $\left(t_{H}-1, t_{D}\right)$ to $\left(t_{H}, t_{D}\right)$ is also by definition beneficial to preference type $H$, given that $t_{H}$ is type $H$ 's best response to $t_{D}$. An equivalent argument shows that preference type $D$ benefits from a transition from $\left(t_{H}-1, t_{D}+1\right)$ to $\left(t_{H}, t_{D}\right)$. First, transiting from $\left(t_{H}-1, t_{D}+1\right)$ to $\left(t_{H}, t_{D}+1\right)$ is beneficial for the preference type $D$ given our assumption that $m>1$. Second, going from $\left(t_{H}, t_{D}+1\right)$ to $\left(t_{H}, t_{D}\right)$ is also by definition beneficial to preference type $D$, given that $t_{D}$ is type $D$ 's best response to $t_{H}$.

Proposition 2 shows that if there exist multiple reactive SSDEs, then the strongly Pareto dominant equilibrium within this set is easily described: it is that in which each preference type acts the least according to its own bias. In other words, it is the equilibrium in which the doves act harshest (have the lowest threshold $t_{D}$ ) and the hawks act the most leniently (have the highest threshold $t_{H}$ ). Reciprocally, the strongly Pareto dominated equilibrium within this set is the one in which preference types act the most in line with their relative bias. Summarizing, as one jumps from the one to the other adjacent equilibrium within the set of reactive SSDEs, the welfare of each type increases, the less that type acts in accordance with its relative bias.

We now finally compare reactive SSDEs with the unique reactive SPDE.

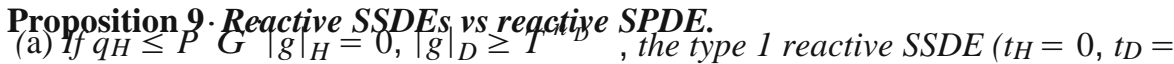
$T_{D}^{n D}$ ) exists and is outcome equivalent to the unique reactive SPDE. Any other

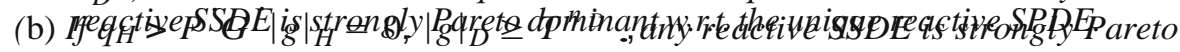
dominant w.r.t. the unique reactive $S B D E$. 
Proposition 3 builds on the following dynamic thought experiment: Start from the unique reactive SPDE, in which doves simply decide as if they were voting alone under

Unanimity, fully pooling their information and optimally coordinating their votes according to the threshold $T_{D}^{n_{D}}$. Now, let hawks Subgroup Deliberate and optimally coordinate their votes under the assumption that doves convict, while doves continue to behave as in the unique reactive SPDE. There are now two possibilities, which are

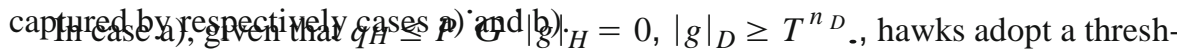

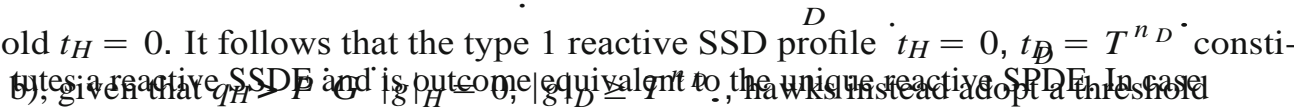
$t_{H}>0$. This adjustment is by definition strictly improving for doves as well, as hawks

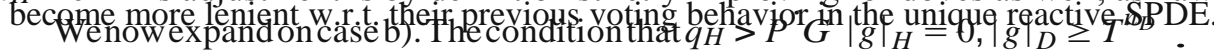

means that the hawks' information is decision relevant in the sense that conditional on ${ }^{\circ}|g|_{H}=0,|g|_{D} \geq T_{D}^{n_{D}}$, hawks favour an acquittal. Clearly, conditional on the information set ${ }^{\circ}|g|_{H}=0,|g|_{D} \geq T_{D}^{n_{D}}$, the above condition implies that a dove would agree that an acquittal is optimal. Consequently, letting doves Subgroup Deliberate and coordinate votes according to $T_{D}^{n_{D}}$, both types gain if hawks now Subgroup Deliberate and coordinate votes according to some optimal threshold $t_{H}>0$ instead of always convicting. Now, let us consider a next round of adjustment: Let the doves optimally readjust their threshold in the light of the threshold $t_{H}$ chosen by hawks in the previous round. It is clear that doves will choose $t_{D} \leq T_{D}^{n}$, so that this adjustment is at least weakly favourable to both preference types. This mutual adjustment process may be continued until a fixed point is reached. Such a fixed point exists if there exists any reactive SSDE (and we know that there indeed exists one), and this fixed point corresponds to the most polarized reactive SSDE. Furthermore given that each step of the considered adjustment process is strongly Pareto improving, this reactive SSDE is strongly Pareto improving w.r.t. the unique reactive SPDE.

As a remark that applies to both cases a) and b) mentioned above, recall that if there exist several reactive SSDEs, we know from Proposition 2 that the most polarized reactive SSDE is strongly Pareto dominated by all remaining reactive SSDEs. It follows that if there are $K>1$ reactive SSDEs, then $K-1$ of these are a priori guaranteed to strongly Pareto dominate the unique reactive SPDE.

We now summarize our welfare comparison of the three protocols. Four cases can be distinguished. The first and least interesting case corresponds to $T_{D}^{n}{ }^{2}=n_{D}$ and

$$
q_{H} \leq P^{\cdot} G^{\cdot}|g|_{H}=0,|g|_{D} \geq T^{n_{D}} .
$$
540 improves on the unique reactive SPDE. The only reactive SSDE that is guaranteed to ${ }_{541}$ exist is outcome equivalent to the unique reactive SNDE and SPDE. 
The second case applies when $T_{D}^{n_{D}}<n_{D}$ while (12) holds. Here, the unique reactive SPDE is strongly Pareto improving w.r.t. to the unique reactive SNDE and the only reactive SSDE of which we can guarantee the existence is outcome equivalent to the unique reactive SPDE. The third case applies when $T_{D}^{n}{ }_{D}=n_{D}$ while (12) is reversed. Here, the unique reactive SPDE is outcome equivalent to the unique reactive SNDE and we know that there exists a reactive SSDE that strongly Pareto improves on the unique reactive SPDE.

The fourth and mostinteresting case applies when $T_{D}^{n_{D}}<n_{D}$ while (12)is reversed. In this case, the unique reactive SPDE is strongly Pareto improving w.r.t. the unique reactive SNDE and we know that there exists a reactive SSDE that strongly Pareto improves on the unique reactive SPDE. We now summarize the intuition for this fourth case. One can think of the stepwise transition from ND to PD and then to SD in terms of two successive improvements. First, as compared to the unique reactive SNDE, the unique reactive SPDE allows an improvement in the aggregation of the doves' information that is beneficial to both preference types. Secondly, as compared to the unique reactive SPDE, reactive SSDEs also allow to use the information held by the hawks, in a way that is advantageous to both preference types.

Given the above propositions, modulo our admittedly restrictive equilibrium selection under the PD and SD protocols, we have thus established a complete ranking of the three protocols considered: Subgroup Deliberation dominates Plenary Deliberation which itself dominates Private Voting. We wish to stress that the suboptimality of the ND protocol w.r.t. the remaining two protocols is a much more robust result than the dominance of SD over PD. Recall indeed that we impose very heavy restrictions on strategy profiles under PD and SD. Our ranking of SD and PD thus remains very tentative.

We close our analysis with two remarks on how our results potentially extend to more general settings. Our first remark concerns the condition $q_{D}>\frac{1}{2}$ imposed throughout. As mentioned already, the condition is key to showing that the $n_{D}$ unique reactive SPDE strongly Pareto dominates the unique reactive SNDE if $T^{n_{D}}<n_{D}$. Now, assuming $T_{D}^{n_{D}}<n_{D}$ and $q_{H}>P^{*} G^{\cdot}|g|_{H}=0,|g|_{D} \geq T_{D}^{n_{D}}$, we conjecture that one can construct examples in which $q_{D}<\frac{1}{2}$ and the following holds true: The unique reactive SPDE is not Pareto improving w.r.t. the unique reactive SNDE, but some reactive SSDE however is. The rationale would be as follows: While the unique reactive SPDE is relatively unattractive in welfare terms, each step of the hypothetical adjustment process leading from the unique reactive SPDE to the most polarized reactive SSDE is Pareto improving and the set of reactive SSDEs is furthermore ordered in the Pareto sense.

\section{Conclusion}

We set out to compare three communication protocols characterized by different physical constraints on information pooling: PD, SD and ND. We identified simple conditions on juror preferences such that the following holds. First, the SD and PD protocols robustly dominate ND in the Pareto sense. The dominance of PD and SD w.r.t ND relies on the fact that the identified reactive SPDE and SSDE allow for a superior 
611 Step 2 We here prove Point a), corresponding to the set of type 2 reactive SSDEs. We 612 first show that the condition given in Point a) is sufficient to ensure that none of the 613 above mentioned two types of deviations is strictly advantageous to a juror of type $614 j$. Assume thus that the condition of Point a) is satisfied. Regarding the first type of 615 mentioned deviation, the threshold adopted by each Subgroup is ex post optimal at the 616 voting stage, conditional on the locally pooled information and assuming individual 617 pivotality, i.e. assuming that that the other Subgroup votes for conviction. We now 618 examine the second type of deviation. Note that misreporting a $g$-signal as an $i$-signal 619 is either inconsequential or adversely triggers an acquittal given a Subgroup signal 620 profile where the deviating juror would have favoured a conviction. This can thus not 621 be strictly advantageous to a juror. Instead, misreporting an $i$-signal as a $g$-signal 622 is always without consequence on the final decision, as a juror can alway block a 623 conviction triggered by his lie if he realizes that he favours acquittal, given remaining 624

aggregation of the information held by doves, in a way that is also beneficial to hawks. Second, to the extent that one focuses on a restricted class of equilibria under PD, class of reactive SSDEs allows to also aggregate the information held by hawks.

Our analysis features a number of restrictions that future research should address. A truly robust comparison of PD and SD would need to characterize the whole set of reactive equilibria under each of the protocols, thus abandonning the restriction to monotonous, symmetric and pure strategies. It may be that PD and SD cannot be ranked in the Pareto sense. One also ought to consider other voting rules than Unanimity. In the case of SD and non unanimous voting rules, we conjecture that welfare
dominant equilibria involve members of the same Subgroup voting asymmetrically. In such equilibria, the number of Subgroup members voting $C$ would increase as a function of the number of $g$-signals held by the Subgroup. Another restriction of our analysis is the unrealistic assumption of only two preference types. Enlarging the set of preference types would however substantially complicate the analysis. One first irection to explore would be to assume that any juror's preference type is located within a neighbourhood of either of two reference values $q_{H}$ or $q_{D}$. Finally, the binary cols ought to be repeated in a setting featuring continuous signals in order to evaluate whether our results still hold in such a more natural and versatile environment.

\section{Appendix 1}

\section{Lemma 2}

Step 1 In a reactive SSDE, two types of individual deviations must be prevented. ment at the communication stage. The second type of deviation involves lying at the communication stage.

\section{Subgroup members' signals.}

We now show that the condition stated in Point a) is necessary to ensure that none of the two types of deviations mentioned in step 1 is strictly advantageous to a juror 
${ }_{627}$ of type $j$. Suppose that thus that the condition is not satisfied. Suppose that $t_{j}$ is larger 628 than specified by the condition, given $t_{-j}$. Then a juror of preference type $j$ has a strict 629 incentive to announce an $i$-signal as a $g$-signal and subsequently vote on the basis of 630 the known signal profile of his Subgroup and the assumption that the other Subgroup 631 convicts. Suppose now instead that $t_{j}$ is smaller than specified by the condition, given ${ }_{632} t-j$. Then a juror of preference type $j$ has a strict incentive to announce a $g$-signal ${ }_{633}$ as an $i$-signal and subsequently vote on the basis of the known signal profile of his 634 Subgroup and the assumption that the other Subgroup convicts.

635 Step 3 We now prove Point b), corresponding to the set of type 1 reactive SSDEs. The 636 analysis of condition (9) for type $j$ follows the exact same steps as in Point a). We now 637 examine condition (10), which applies to the type that always convicts independently 638 of the its Subgroup signal profile. Note first that a juror of type $-j$ must be willing to 639 convict no matter what signal profile is revealed at the communication stage, which 640 requires (10) to hold. This proves that (10) is necessary. We now show that condition

641 (10) is sufficient to ensure no strict incentive to deviate for type $-j$. An individual 642 of type $-j$ recognizes that his announced signal is inconsequential for the voting 643 behavior of his Subgroup and thus has no incentive to deviate from truthtelling. As to 644 the voting stage, conviction is always ex post optimal, assuming individual pivotality, 645 i.e. assuming that that the other Subgroup votes for conviction. It follows that a type $646-j$ has no strict incentive to deviate at the voting stage.

${ }_{647}$ Step 4 In the next steps, we show that our characterization of the set of reactive ${ }_{648} \mathrm{SSDEs}$ generalizes to a larger set of voting rules. Let $R$ be the minimal number of 662 identified in step 1 is strictly advantageous. Assume thus that condition of Point a) is 663 respected. Regarding the first type of mentioned deviation, the threshold adopted by 664 each Subgroup is ex post optimal at the voting stage, conditional on the locally pooled 665 information and assuming individual pivotality, i.e. assuming that that the other Sub666 group votes for conviction. We now examine the second type of deviation. Note that 667 misreporting a $g$-signal as an $i$-signal is either inconsequential or adversely triggers 668 an acquittal given a signal profile where the deviating juror would have favoured a

conviction votes required for a conviction decision and assume that $R>\left\{n_{H}, n_{D}\right\}$.

Two key aspects deserve mention. First, assuming $R>\left\{n_{H}, n_{D}\right\}$ means that individual pivotality, either in communicating or in voting, implies that the Subgroup to which one does not belong votes for conviction. This replicates the case of Unanimity. A second key aspect is that abandoning Unanimity implies that an individual can now not single handedly veto a conviction anymore. Accordingly, deviating to announcing a $g$-signal when holding an $i$-signal is now risky, in the sense that one cannot simply veto an undesirable collective conviction vote triggered by such a deviation. We now show that the necessary and sufficient conditions given for the case of Unanimity, whether in Point a) or Point b), extend to this more general case.

Step 5 We first look at the set of type 2 reactive SSDEs. We first show that the condition of Point a) is sufficient to ensure that none of the two types of deviations conviction. This can thus not be strictly advantageous to a juror. Instead, misreporting an $i$-signal as a $g$-signal is either inconsequential or adversely triggers a conviction 
given a signal profile where the deviating juror would have favoured an acquittal. This can thus not be strictly advantageous to a juror.

We now show that the condition given in Point a) is necessary to ensure that none of the two types of deviations mentioned in step 1 is strictly advantageous. Suppose thus that the condition is not satisfied. Suppose that $t_{j}$ is larger than specified by the condition, given $t_{-j}$. Then a juror of preference type $j$ has a strict incentive to announce an $i$-signal as a $g$-signal and subsequently vote on the basis of the known signal profile of his Subgroup and the assumption that the other Subgroup convicts. Suppose that instead $t_{j}$ is smaller than specified by the condition, given $t_{-j}$. Then a juror of preference type $j$ has a strict incentive to announce a $g$-signal as an $i$-signal and subsequently vote on the basis of the known signal profile of his Subgroup and the assumption that the other Subgroup convicts.

${ }_{683}$ Step 6 We now examine the set of type 1 reactive SSDEs. The analysis of (9) for type ${ }_{684} j$ follows the exact same steps as the analysis of type 2 reactive SSDEs. The analysis

685 of (10), corresponding to type $-j$, is identical to that given in step 3 and thus not repeated.

\section{A further lemma on reactive SSDEs}

The following lemma states in close form the existence conditions for a type 2 reactive SSDE.

\section{Lemma 10 SSDEs.}

$\left(t_{H}, t_{D}\right)$ constitutes a type 2 reactive $S S D E$ iff, $\forall j \in\{H, D\}$, it holds that $t_{j} \in$ ${ }^{-} 1, \ldots, n_{j}{ }^{-}$and

$$
\frac{F\left(p, q_{j}\right)+n_{j}+\mathrm{K}^{\cdot} p, t_{-j}, n_{-j}}{2}<t_{j} \leq \frac{F\left(p, q_{j}\right)+n_{j}+\mathrm{K}^{\cdot} p, t_{-j}, n_{-j}+2}{2},
$$

where

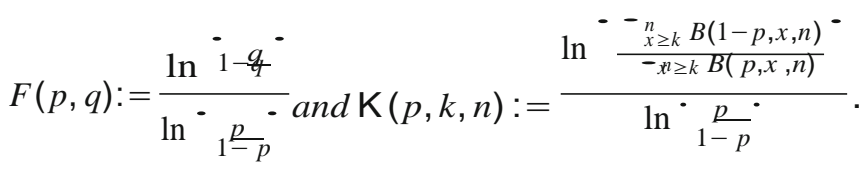

Proof Note that $\left(t_{H}, t_{D}\right)$ constitutes a type 2 reactive SSDE iff, $\forall j \in\{H, D\}$, it holds that $t_{j} \in{ }^{-} 1, \ldots, n_{j}{ }^{-}$and the following two inequalities simultaneously hold:

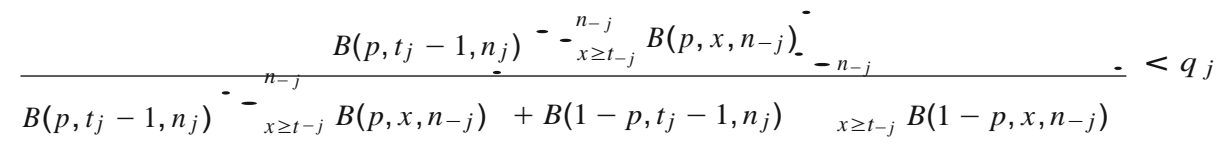


714 Step 1 We first analyze the set of reactive SNDEs in which both preference types 715 condition their play on their information. Note that a given preference type cannot mix 716 after both $i$ - and $g$-signals (see Condition 4). Within this subclass of equilibria, there 717 are altogether nine possible symmetric voting profiles which are listed and numbered

Table 1

\begin{tabular}{lllllllll}
\hline & $\sigma_{g}^{H}, \sigma_{i}^{H}$ & $\sigma_{g}^{D}, \sigma_{i}^{D}$ & & $\sigma_{g}^{H}, \sigma_{i}^{H}$ & $\sigma_{g}^{D}, \sigma_{i}^{D}$ & & $\sigma_{g}^{H}, \sigma_{i}^{H}$ & $\sigma_{g}^{D}, \sigma_{i}^{D}$ \\
\hline $\mathbf{1}$ & 1,0 & 1,0 & $\mathbf{4}$ & $x, 0$ & 1,0 & $\mathbf{7}$ & $x, 0$ & $1, y$ \\
$\mathbf{2}$ & 1,0 & $x, 0$ & $\mathbf{5}$ & $1, x$ & 1,0 & $\mathbf{8}$ & $1, x$ & $y, 0$ \\
$\mathbf{3}$ & 1,0 & $1, x$ & $\mathbf{6}$ & $x, 0$ & $y, 0$ & $\mathbf{9}$ & $1, x$ & $1, y$ \\
\hline
\end{tabular}

Applying the ln-transformation to both sides of (17), the above inequality can then be rewritten as follows:

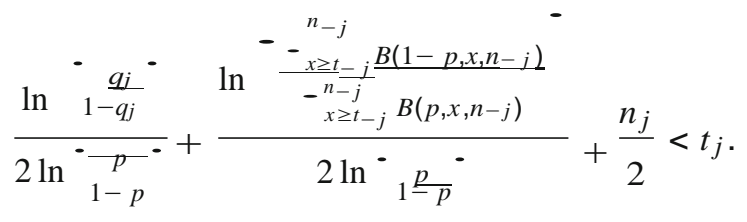

One can perform a similar transformation for (16). One obtains an inequality stating that $t_{j}$ is weakly smaller than the LHS expression in (18) plus one.

\section{Appendix 2}

\section{Lemma 4: reactive SNDEs} in Table 1 below. Letters $x, y \in(0,1)$ are used to denote mixing probabilities. 
719 720 721

We show that none of the above nine strategy profiles constitutes an equilibrium. Equilibrium 1 trivially never exists when $m>1$. Equilibria 2,4 and 6 do not exist under the assumption that $q_{D}<\beta(p, n, n)$ given that they require either $q_{D}=\beta(p, n, n)$ or $q_{H}=\beta(p, n, n)$ (recall $\left.q_{H}<q_{D}\right)$. Recall in what follows that $p i \vee_{j}$ stands for the event in which a juror of preference type $j$ is pivotal, i.e. all remaining jurors vote for conviction. Equilibria 3,7 and 9 imply (19) and (20), as given below.

$$
\begin{aligned}
& q_{D}=P\left(G \mid i, p i \mathrm{v}_{D}\right) \\
& (1-p) p \sigma_{g}^{D}+(1-p) \sigma_{i}^{D}{ }_{g}^{-n_{D}-1} p \sigma_{g}^{H}+(1-p) \sigma_{i}^{H^{-n_{H}}}
\end{aligned}
$$

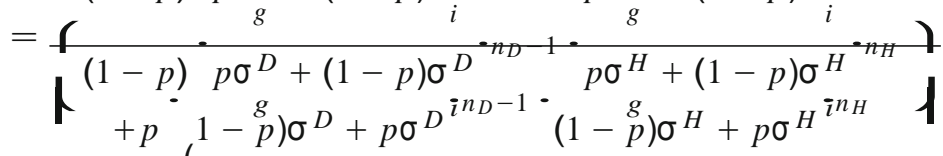

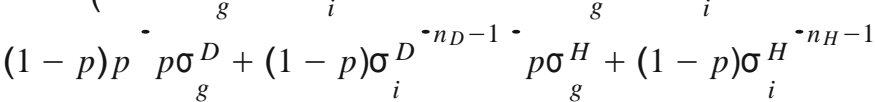

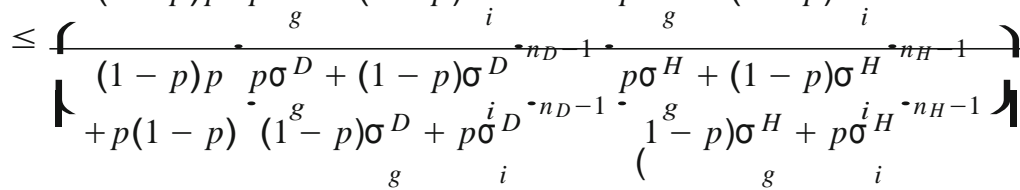

$$
\begin{aligned}
& =\frac{p F_{p}^{1}}{p F_{p}^{1}+(1-p) F_{1-p}^{1}}=: \overline{P_{1}} \\
& q_{H} \geq P\left(G \mid i, p i \mathrm{v}_{H}\right) \\
& (1-p) p \sigma_{g}^{D}+(1-p) \sigma_{i}^{D} \quad p \sigma_{g}^{H}+(1-p) \sigma_{i}^{{ }^{-n_{D}}}{ }^{-n_{H}-1}
\end{aligned}
$$

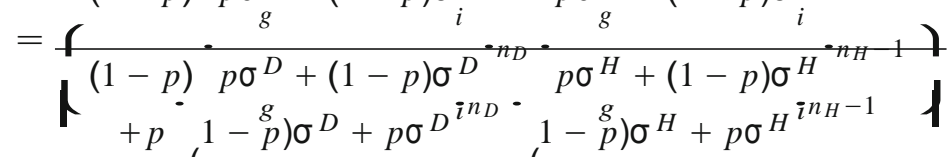

$$
\begin{aligned}
& (1-p)^{2} p \sigma_{g}^{D}+(1-p) \sigma^{D}{ }^{-n_{D}-1} \cdot \frac{g}{p \sigma^{H}}+(1-p) \sigma_{i}^{H}{ }^{-n_{H}-1} \\
& \left.>\mathbf{(}(1-p)^{2} \cdot p \sigma^{D}+(1-p) \sigma^{D}{ }^{-n_{D-1}} \cdot p \sigma^{H}+(1-p) \sigma^{H^{-n_{H}}-1}\right) \\
& +p^{2}(1-\stackrel{g}{p}) \sigma^{D}+p \sigma^{D}{ }_{i}^{i^{i n_{D}-1}}(1-\stackrel{g}{p}) \sigma^{H}+p \sigma^{H^{i n_{H}-1}} \\
& =\frac{(1-p) F_{p}^{1}}{(1-p) F_{p}^{1}+p F_{1-p}^{1}}=: \underline{P}_{1} \text {, }
\end{aligned}
$$

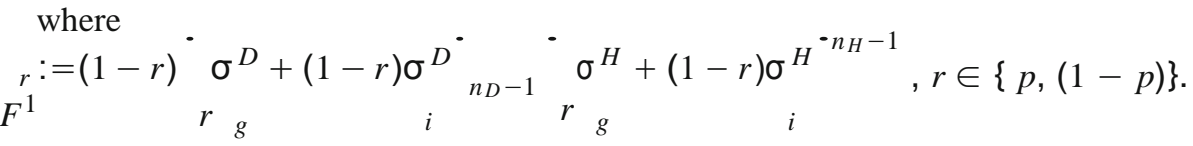

Now, using the fact that for any positive constants $A, B, C, D,{ }_{C}^{A}{ }_{+}^{A}-\frac{C}{C+D} \Leftrightarrow$ ${ }_{B}^{A} \leq{ }_{D}$, note that there exists a positive integer $T$ s.t. 


$$
\begin{aligned}
\frac{B(p, T-1, n)}{B(1-p, T-1, n)} & =\frac{p^{T-1}(1-p)^{n-T+1}}{(1-p)^{T-1} p^{n-T+1}} \leq \underbrace{(1-p) F_{p}^{1}}_{p F_{1-p}^{1}} \leq \frac{p^{T}(1-p)^{n-T}}{(1-p)^{T} p^{n-T}} \\
& =\frac{B(p, T, n)}{B(1-p, T, n)}
\end{aligned}
$$

and multiplying all expressions $\frac{b y p^{2}}{(1-p)^{2}}$

$$
\begin{aligned}
\frac{B(p, T, n)}{B(1-p, T, n)} & =\frac{p^{T}(1-p)^{n-T} \overline{(1-p)^{2}}}{(1-p)^{T} p^{n-T}} \leq \frac{p F^{1}}{(1-p) F_{1-p}^{1}} \leq \frac{p^{T+1}(1-p)^{n-T-1}}{(1-p)^{T+1} p^{n-T-1}} \\
& =\frac{B(p, T+1, n)}{B(1-p, T+1, n)} .
\end{aligned}
$$

Summarizing, inequalities (19) and (20) thus imply that there exists a positive integer $T$ s.t.:

$$
\beta(p, T-1, n) \leq \underline{P}_{1} \leq q_{H}<q_{D} \leq \overline{P_{1}} \leq \beta(p, T+1, n) .
$$

The inequality relation (23) however means that $m \leq 1$ if equilibrium 3,7 or 9 exist. But we have assumed $m>1$. As to equilibria 5 and 8 , note that they imply that the following two conditions (24) and (25) hold:

49

$$
\begin{aligned}
& q_{H}=P\left(G \mid i, p i \mathrm{v}_{H}\right) \text {. }
\end{aligned}
$$

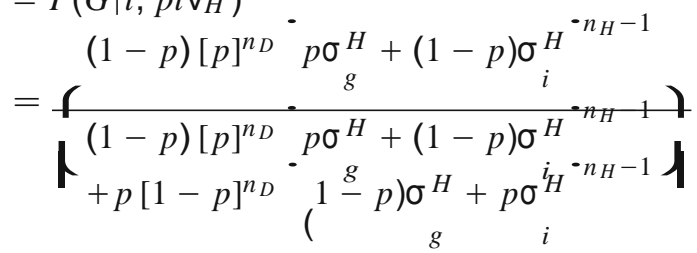$$
=\frac{(1-p) F_{p}^{2}}{(1-p) F_{p}^{2}+p F_{1-p}^{2}}=: \underline{P_{2}},
$$

$$
\begin{gathered}
q_{D} \leq P\left(G \mid g, p i \mathrm{v}_{D-}\right) \\
{[p]^{n_{D}} p \sigma_{g}^{H}+(1-p) \sigma_{i}^{H^{-n_{H}}}}
\end{gathered}
$$



760 assumed $m>1$. To summarize Step 1, we have now shown that none of the nine 761 possible reactive SND voting profiles in which both types condition their play on their 762 information (as listed in Table 1) ever constitutes an equilibrium.

763 Step 2The next steps examine the set of putative reactive SNDEs in which at least one of 764 the two preference types plays $\left(\sigma_{g}=1, \sigma_{i}=1\right)$ while the other type conditions its play 765 on its information. Here, altogether six profiles need to be considered, depending on 766 the nature of the strategy, $\left(\sigma_{g}=1, \sigma_{i}=0\right)$ or $\left(\sigma_{g}=1, \sigma_{i}=x\right)$ or $\left(\sigma_{g}=y, \sigma_{i}=0\right)$, ${ }_{767} 0<x, y<1$, played by the preference type that conditions its play on its signal

where

The inequalities (24) and (25) imply that there exists a positive integer $T$ s.t.:

$$
\beta(p, T-1, n) \leq \underline{P}_{2}=q_{H}<q_{D}<\overline{P_{2}} \leq \beta(p, T+1, n) .
$$
as well as on the identity of the concerned preference type. Step 3 deals with the set of putative equilibrja in which the hawks condition their play on their information while doves play $\left(\sigma=1, \sigma D_{i}=1\right)$. We show that this set is empty. Step 4 examines ( $\sigma \stackrel{\text { equilibria in which the doves condition play on their signals while the hawks play }}{=} 1, \sigma^{H}=1$ ). $\left.g \quad i_{i}=1\right)$.

Step 3 . We here examine strategy profiles in which the hawks condition their play on their signal while the doves play $\left.\underset{g}{\left(\sigma^{D}\right.}=1, \sigma_{i}^{D}=1\right)$. In such an equilibrium it must be the case that:

$$
\begin{aligned}
& P\left(G \mid i, p i \vee_{H}\right) \leq q_{H} \leq P\left(G \mid g, p i \vee_{H}\right) \\
& q_{D} \leq P\left(G \mid i, p i \vee_{D}\right)<P\left(G \mid g, p i \vee_{D}\right)
\end{aligned}
$$

Now, note however that:

$$
\begin{aligned}
& P\left(G \mid i, p i \mathrm{\vee}_{H}\right) \quad(1-p)^{-} p \sigma^{H}+(1-p) \sigma^{H^{-n_{H-1}}}
\end{aligned}
$$

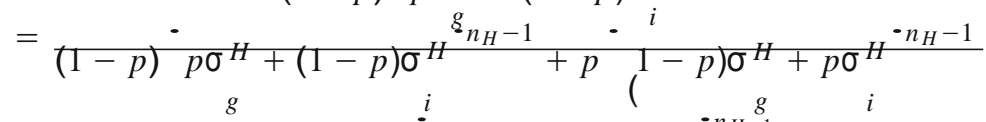

$$
\begin{aligned}
& (1-p)^{2} \stackrel{i}{i} p \sigma_{g}^{H}+(1-p) \sigma_{i}^{H}{ }^{-n_{H-1}}
\end{aligned}
$$

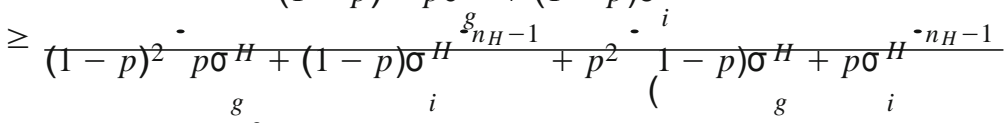

$$
\begin{aligned}
& =\frac{(1-p) F_{p}^{3}}{(1-p) F_{p}^{3}+p F_{1-p}^{3}}=: \underline{P} \text {, }
\end{aligned}
$$




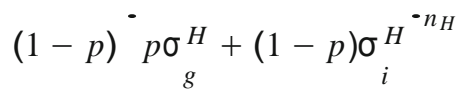

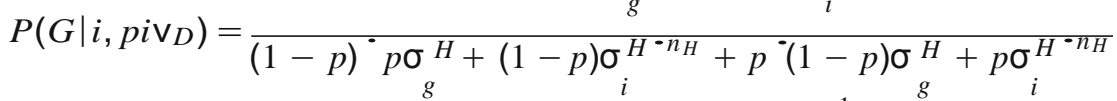

$$
\begin{aligned}
& (1-p) p \quad p \sigma^{H}+(1-p) \sigma^{H^{-n_{H}-1}}
\end{aligned}
$$

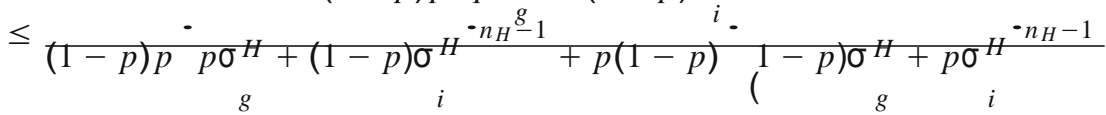

$$
\begin{aligned}
& =\frac{p F_{p}^{3}}{p F_{p}^{3}+(1-p) F_{1-p}^{3}}=: \overline{P_{3}},
\end{aligned}
$$

where

$$
F^{3}:=(1-r){ }_{r}^{\bullet} \sigma_{i}^{H}+(1-r) \sigma^{H^{-}} n_{H-1}, r \in\{p,(1-p)\} .
$$

Now, (29) and (30) imply that there exists a positive integer $T$ s.t.:

$$
\beta(p, T-1, n) \leq \underline{P}_{3} \leq q_{H}<q_{D} \leq \overline{P_{3}} \leq \beta(p, T+1, n) .
$$

This in turn means that $m \leq 1$. We have however assumed $m>1$. Therefore this type of equilibria does not exist.

Step 4 We now examine equilibria in which the doves condition play on their signals while the hawks play $\left(\sigma^{H}=1, \sigma{ }^{H}=1\right)$. There are a priori three such candidates.

The first candidate is the equilibrium given by $\left(\sigma_{g}{ }^{H}=1, \sigma_{i}{ }^{H}=1, \sigma_{g}{ }^{D}=x, \sigma_{i}{ }^{D}=0\right)$, for $0<x<1$. However, it exists iff $q_{D}=\beta\left(p, n_{D}, n_{D}\right)$, which is never true by assumption. The second candidate is the putative equilibrium A given by $\left(\sigma^{H}{ }_{g}=\right.$ $\left.1, \sigma_{i}^{H}=1, \sigma_{g}^{D}=1, \sigma_{i}^{D}=0\right)$. The third candidate is the putative equilibrium $\mathrm{B}$ given by $\left(\sigma_{g}^{H}=1, \sigma_{i}^{H}=1, \sigma_{g}^{D}=1, \sigma_{i}^{D}=y\right)$, for $0<y<1$. We show that either equilibrium A or B (never both) exists for any $q_{D} \in\left((1-p), \beta\left(p, n_{D}, n_{D}\right)\right)$. Equilibrium A trivially exists iff $\beta\left(p, n_{D}-1, n_{D}\right)<q_{D}<\beta\left(p, n_{D}, n_{D}\right)$. As to equilibrium $\mathrm{B}$, note that $y$ satisfies:

$$
q_{D}=\frac{(1-p)[p+(1-p) y]^{n_{D}-1}}{(1-p)[p+(1-p) y]^{n_{D}-1}+p[1-p+p y]^{n_{D}-1}},
$$

so that, recalling explicitly the dependence of $y$ on $p, q_{D}$ and $n_{D}$,

$$
y\left(p, q_{D}, n_{D}\right)=\frac{{\frac{\left(1-q_{D}\right)(1-p)}{q_{D} p}}^{-\frac{1}{n_{D}-1}} p-(1-p)}{p-{\frac{\left(1-q_{D}\right)(1-p)}{q_{D}}}^{-{ }^{n_{D}-1}}(1-p)} .
$$


${ }_{813}$ Step 1 Suppose a reactive SPDE in which hawks trutfully reveal their signals and 814 doves babble. We know from Lemma 3 that such an equilibrium exists iff there 832 833

Now, note that $y\left(p, 1-p, n_{D}\right)=1, y\left(p, \beta\left(p, n_{D}-1, n_{D}\right), n_{D}\right)=0$ and

$$
\frac{\partial y\left(p, q_{D}, n_{D}\right)}{\partial q_{D}}
$$$$
=
$$

$$
p q_{D}^{2}\left(n_{D}-1\right) \quad p-{ }_{p q_{D}}(p-1)\left(q_{D}-1\right) \bar{D}+p-\bar{p} q_{D}(p-1)\left(q_{D}-1\right)
$$

is a $t_{H} \in\left\{1, \ldots, n_{H}\right\}$ s.t. $\beta\left(p, t_{H}-1, n_{H}\right)<q_{H} \leq \beta\left(p, t_{H}, n_{H}\right)$ and $q_{D} \leq$ $\beta\left(p, t_{H}, n_{H}+1\right)$. However, given our assumption that $m>1$, there by definition exists no such $t_{H}$.

Step 2 Suppose now a reactive SPDE in which doves truthfully reveal their signals and hawks babble. Given our assumption on $q_{D}$, there exists a (unique) $t^{*} \in\left\{1, \ldots, n_{D}\right\}$ s.t. $\beta^{\circ}, t_{D}^{*}-1, n_{D}<q_{D} \leq \beta^{*} \underset{D}{t}{ }^{*}, n_{D} \cdot$. Furthermore, we know that $q_{H} \leq$ $\beta p, t_{D}^{*}, n_{D}+1^{*}$ given our assumption that $m>1$. It follows from Lemma 3 that there exists a unique SPDE in which doves truthfully communicate while hawks babble.

\section{Lemma 6: reactive SSDEs}

Point a) Note first that there exists a type 2 reactive SSDE if :

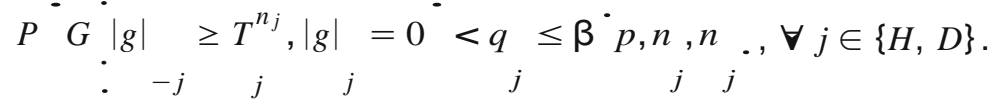

Note that there exists a type 1 reactive SSDE given by $t_{j} \in{ }^{-} 1, \ldots, n_{j}{ }^{-}$and $t_{-j}=0$ iff:

$$
\beta^{\cdot} p, 0, n_{j}<q_{j} \leq \beta^{\cdot} p, n_{j}, n_{j}{ }^{\circ} \cap q_{-j} \leq\left. P^{-} G^{\cdot}|g|\right|_{j} \geq T_{j}{ }^{n_{j}},|g|_{-j}=0^{\cdots} \text {. }
$$

Clearly, using together conditions (34) and (35), there always exists some reactive SSDE given our assumptions on $q_{H}$ and $q_{D}$. Indeed, if $\beta\left(p, 0, n_{H}\right)<q_{H}<$ $\beta\left(p, n_{H}, n_{H}\right)$ and $\beta\left(p, 0, n_{D}\right)<q_{D}<\beta\left(p, n_{D}, n_{D}\right)$, then either (34) is true or (35) is true for some $j \in\{H, D\}$. Note finally that conditions (34) and (35) do not 

839 it is readily checked that there exist two type 2 reactive SSDEs given by respectively $840\left(t_{H}=3, t_{D}=4\right)$ and $\left(t_{H}=2, t_{D}=5\right)$.

841 Point b) Using the conditions given in Lemma 7 in Appendix 1, call $t_{i}^{B R}\left(t_{j}\right)$ the

${ }_{844}$ Suppose that $(k, l)$ constitutes a reactive SSDE. Given the behavior of $t{ }_{D}^{B R}\left(t_{H}\right)$, only 845 the four following threshold profiles may also constitute reactive SSDEs: $(k-1, l+1)$, ${ }_{846}(k-1, l),(k+1, l)$ or to $(k+1, l-1)$. Furthermore, given the behavior of $t_{H}^{B R}\left(t_{D}\right)$, only 847 the four following threshold profiles may also constitute reactive SSDEs: $(k-1, l+1)$, $848(k, l+1),(k, l-1)$ or $(k+1, l-1)$. Taking the intersection of the two sets, the only 849 neighbouring points to $(k, l)$ that may constitute reactive SSDEs are $(k-1, l+1)$ or

prohibit the simultaneous existence of a type 1 reactive SSDE and a type 2 reactive SSDE.

Note that there may exist multiple reactive SSDEs. We prove this by an example. unique best response threshold of Subgroup $i$ to the threshold $t j_{i}$ of Subgroup
defined in (13). Note that either $t_{i}^{B R}\left(t_{j}+1\right)=t_{i}^{B R}\left(t_{j}\right) \underset{i}{\text { or }} t^{B R}\left(t_{j}+1\right) \underset{i}{=} t^{B R}\left(t_{j}\right)-1$. $(k+1, l-1)$. Suppose finally that the two best response functions do not intersect in any of these two neighbouring points. Then, this implies that they do not intersect in any other point than $(k, l)$.

\section{Appendix 3}

\section{Proposition 1: reactive SPDE vs reactive SNDE}

Step 1 Recall that the unique reactive SPDE involves doves truthfully revealing their signal and voting according to $T_{D}^{n} D$ while hawks babble and always convict.

Step 2 Recall that there always exists a unique reactive SNDE, given by profile A or B. Recall also that profile $A$ is given by $\left(\sigma_{g}=1, \sigma_{i}^{H}=1, \sigma \underset{g}{=}=1, \sigma=0\right)$. Suppose that $\beta\left(p, n_{D}-1, n_{D}\right)<q_{D}<\beta\left(p, n_{D}, n_{D}\right)$, so that equilibrium $\mathrm{A}$ is the unique reactive SNDE. For these parameter values, the unique reactive SNDE and the unique reactive SPDE are thus outcome equivalent.

Step 3 Steps 3 to 9 are dedicated to the examination or parameter values for which

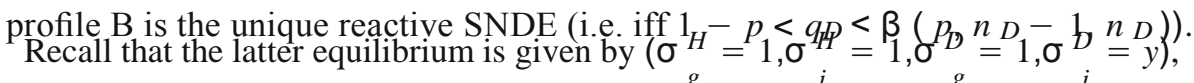
with $y \in(0,1)$. The unique reactive SPDE is here characterized by a dove threshold $T B^{D} \leq n_{D}-1$. The transition from the unique reactive SNDE to the unique SPDE is clearly strictly beneficial to the doves, as these are now optimally aggregating their information. In contrast, it however remains unclear whether the transition from the first to the second equilibrium is strictly beneficial to the hawks as well. If we can prove that this is the case, then we know that the unique reactive SPDE is strongly Pareto improving w.r.t to the unique reactive SNDE, for the concerned parameter values.

Step 3 All we need is thus to show that, starting from the reactive SND profile B, allowing doves to Subgroup Deliberate while keeping the hawks' play fixed will be strictly beneficial to the hawks. We do so in the next steps. Denote by $\mathrm{M}_{j}\left(q_{D}, S D, t_{D}\right)$ 
876 the expected payoff of preference type $j$ when the doves are allowed to Subgroup ${ }_{877}$ Deliberate and adopt a threshold $t_{D}$, while hawks always all vote for conviction as in

\section{5} 896 able to share their information when they Subgroup Deliberate, $W\left(q_{D}, q_{D}\right)>0$. If 897 we can show that for all values of $q_{D}$ and corresponding values $t_{D}\left(q_{D}\right)$ and $y\left(q_{D}\right)$, 898 the derivative $\partial W\left(q_{j}, q_{D}\right) / \partial q_{j}$ is negative, then it is also true that $W\left(q_{H}, q_{D}\right)>0$, the reactive SND profile B. Let $t_{D}\left(q_{D}\right)$ be the optimal threshold adopted by the doves in these circumstances, given $q_{D}$, i.e. let $t_{D}\left(q_{D}\right)=T_{D}^{n_{D}}$. Denote by $\mathrm{M}_{j}\left(q_{D}, N D\right)$ the expected payoff of preference type $j$ in the reactive SND equilibrium B. Denote by $y\left(q_{D}\right)$ the mixing probability of the doves after an $i$-signal in the reactive SND equilibrium B. Note that:

$$
\begin{aligned}
& W\left(q_{j}, q_{D}\right):=\mathrm{M}_{j}\left(q_{D}, \underset{n_{D}}{S D}, t_{D}\left(q_{D}\right)\right)-\mathrm{M}_{j}\left(q_{D}, N D\right) \\
& =-P(G)-B\left(p, x, n_{D}\right)\left[y\left(q_{D}\right)\right]^{n_{D}-x}\left(1-q_{j}\right) \\
& x=0 \\
& +P(I)-B\left(1-p, x, n_{D}\right)\left[y\left(q_{D}\right)\right]^{n_{D}-x} q_{j} \\
& x=0 \\
& +P(G) \underset{x=t_{D}\left(q_{D}\right)}{-} B\left(p, x, n_{D}\right)\left(1-q_{j}\right) \\
& \text { nD } \\
& -P(I) \underset{x=t_{D}\left(q_{D}\right)}{-} B\left(1-p, x, n_{D}\right) q_{j} .
\end{aligned}
$$

It follows that:

$$
\begin{aligned}
& \partial \underset{j}{W}(q, q) / \partial q \underset{j}{\stackrel{1}{=}} \stackrel{n_{D}-}{2} \underset{x^{\prime}=0}{n_{D}}\left(B\left(p, x, n_{D}\right)+B\left(1-p, x, n_{D}\right)\right)\left[y\left(q_{D}\right)\right]^{n_{D}-x} \\
& -\overline{2}_{x=t_{D}\left(q_{D}\right)}\left(B\left(p, x, n_{D}\right)+B\left(1-p, x, n_{D}\right)\right) .
\end{aligned}
$$

The sign of $\partial W\left(q_{j}, q_{D}\right) / \partial q_{j}$ is thus determined by the difference in the total robability of conviction implied by each of the two voting scenarios considered, i.e.

No Deliberation by the doves according to the symmetric voting strategy $\left(\sigma{ }_{g}^{D}=1\right.$, $\left.\sigma_{i} D=y\left(q_{D}\right)\right)$ or Subgroup Deliberation by the doves with an optimally chosen conviction threshold $t_{D}\left(q_{D}\right)$. As the hawks' strategy is unchanged and the doves are because $q_{H}<q_{D}$. Which in other words means that also the hawks benefit from the change in the doves' strategy, if they continue to apply the strategy $\left.\underset{g}{(\sigma} H=\underset{i}{1, \sigma} H^{\prime}=1\right)$ that they follow in the reactive SND equilibrium B.

Step 4 Define the following two expressions:

$$
I\left(n_{D}\right)=\frac{n_{D}}{2}+1 \text { if } n_{D} \text { is even; }=\frac{n_{D}+1}{2} \text { if } n \text { is uneven. }
$$


and for all $z \in\left\{I\left(n_{D}\right), \ldots, n_{D}\right\}$

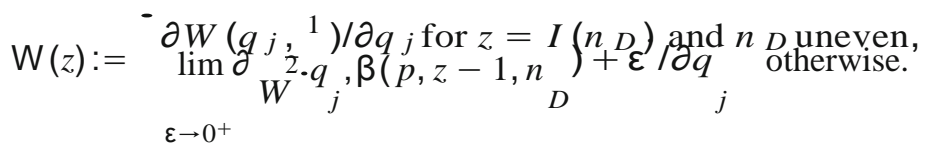

906 In order to show that $\partial W\left(q_{j}, q_{D}\right) / \partial q_{j}$ is negative for all $q_{D} \in{ }^{\bullet} \overline{2}^{1}, \beta\left(p, n_{D}-1\right.$, $\left.907 n_{D}\right)$ ), it is enough to verify that $\mathrm{W}(z) \leq 0$, for all $z \in\left\{I\left(n_{D}\right), \ldots, n_{D}\right\}$. This 908 is true for the two following reasons. First, stating that $W(z) \leq 0$, for all $z \in$ $909\left\{I\left(n_{D}\right), \ldots, n_{D}\right\}$ is equivalent to stating that $\partial W\left(q_{j}, q_{D}\right) / \partial_{j} \leq 0$ for $q_{D}=\frac{1}{2}$ 910 as well as for $q_{D}=\lim _{\varepsilon \rightarrow 0^{+}} \beta\left(p, z-1, n_{D}\right)+\varepsilon, \forall z \in\left\{I\left(n_{D}\right)+1, \ldots, n_{D}\right\}$. Sec911 ondly, given that $y\left(q_{D}\right)$ is decreasing in $q_{D}$ and given that $t_{D}\left(q_{D}\right)$ is constant for all ${ }_{912} q_{D} \in\left(\beta\left(p, z-1, n_{D}\right), \beta\left(p, z, n_{D}\right)\right]$, the derivative $\partial W\left(q_{j}, q_{D}\right) / \partial q_{j}$ is a decreasing 913 function of $q_{D}$ for all $q_{D} \in\left(\beta\left(p, z-1, n_{D}\right), \beta\left(p, z, n_{D}\right)\right]$.

914 Step 5 The proof that $\mathrm{W}(z) \leq 0$ for all $z \in\left\{I\left(n_{D}\right), \ldots, n_{D}\right\}$ is divided into five steps $915\left(6,7,8,9\right.$ and 10). Step 6 shows that $\mathrm{W}\left(n_{D}\right) \leq 0$. Step 7 shows that $\mathrm{W}\left(I\left(n_{D}\right)\right) \leq 0$, 916 for all $n_{D}$ even. Step 8 shows that $\mathrm{W}\left(I_{(}\left(n_{D}\right)\right) \leq 0$ and $\mathrm{W}\left(I\left(n_{D}\right)+1\right) \leq 0$, for all $917 n_{D}$ uneven. Step 8 shows the following. If $n_{D}$ is even, then if $\mathrm{W}(z) \leq \mathrm{W}(z+1)$, it ${ }_{918}$ follows that $\mathrm{W}(z+1) \leq \mathrm{W}(z+2)$ for all $z \in\left\{I\left(n_{D}\right), \ldots, n_{D}-1\right\}$. If, in contrast, ${ }_{919} n_{D}$ is uneven, then if $\mathrm{W}(z) \leq \mathrm{W}(z+1)$, it follows that $\mathrm{W}(z+1) \leq \mathrm{W}(z+2)$ for 920 all $z \in\left\{I\left(n_{D}\right)+1, \ldots, n_{D}-1\right\}$. Step 10 , finally, shows that the four facts proven in 921 steps 6, 7, 8 and 9 imply together that $\mathrm{W}(z) \leq 0$, for all $z \in\left\{I\left(n_{D}\right), \ldots, n_{D}\right\}$.

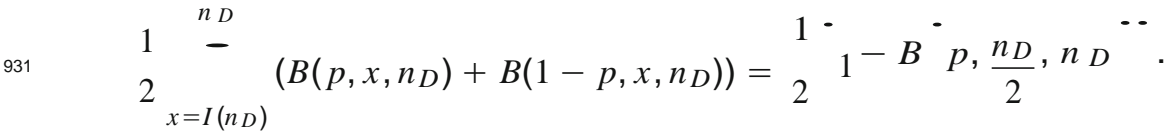

Fact $1: \mathrm{W}\left(n_{D}\right)<0$ whether $n_{D}$ is even or uneven.

Setting $z=n_{D}$, Fact 1 follows immediately from the fact that $y\left(\beta\left(p, n_{D}-1, n_{D}\right)\right)$ $=0$ while $\lim t_{D}\left(\beta\left(p, n_{D}-1, n_{D}\right)+\varepsilon\right)=n_{D}$. $\varepsilon \rightarrow 0^{+}$

Step 7 Note the following fact:

Fact $2: \mathrm{W}\left(I\left(n_{D}\right)\right)<0$ if $n_{D}$ is even.

${ }_{1}$ Note here that $\beta\left(p, I\left(n_{D}\right)-1, n_{D}\right)=\frac{1}{2}$. Also, $t_{D}\left(q_{D}\right)=I\left(n_{D}\right)$ if $q_{D} \in$ $\left({ }_{1}^{1}, \beta\left(p, I\left(n_{D}\right), n_{D}\right)\right)$. For $t_{D}\left(q_{D}\right)=I\left(n_{D}\right)$, the total probability of conviction, if doves Subgroup Deliberate and hawks always convict, is given by:

On the other hand, for $q_{D}={ }_{2}$, the total probability of conviction in the equilibrium $\mathrm{B}$ is given by: 


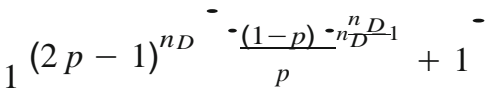

$$
\begin{aligned}
& 2^{-} p-\frac{(1-p)}{p}{ }^{-\frac{1}{n_{D}}}-1 \quad \begin{array}{r}
-n_{D} \\
.
\end{array}
\end{aligned}
$$

935 Now, note that $(42) \leq(41)$, for any $p>\frac{1}{2}$ and $n_{D} \geq 4$. Note that given that we ${ }_{936}$ impose $q_{D}>{ }^{1}$, the equilibrium B does not exist if $n_{D}=2$ so that we can ignore this case. Indeed, B exists only if $q_{D}<\beta\left(p, n_{D}-1, n_{D}\right)$. For the case of $n_{D}=2$, this translates into $q_{D}<\beta(p, 1,2)=\frac{1}{2}$ which contradicts the assumption that $q_{D}>\frac{1}{2}$.

Step 8 Note the following fact:

$$
\text { Fact } 3: \mathrm{W}\left(I\left(n_{D}\right)\right)<0 \text { and } \mathrm{W}\left(I\left(n_{D}\right)+1\right)<0 \text { if } n_{D} \text { is uneven. }
$$

We first look at W $\left(I\left(n_{D}\right)\right)$. For $q_{D}=\frac{1}{2}$ note that $t_{D}\left(q_{D}\right)=I\left(n_{D}\right)$. The total probability of conviction for $t_{D}\left(q_{D}\right)=I\left(n_{D}\right)$, if doves Subgroup Deliberate and hawks always convict, is givenby:

$$
2_{x=I\left(n_{D}\right)}^{1}-\left(B\left(p, x, n_{D}\right)+B\left(1-p, x, n_{D}\right)\right)=\frac{1}{2} .
$$

On the other hand, for $q_{D}=\frac{1}{2}$, the total probability of conviction in the equilibrium $\mathrm{B}$ is given by:

$$
\begin{aligned}
& 1(2 p-1)^{n_{D}} \frac{-(1-p)^{-} n^{n} D_{D} D_{1}}{p}+1
\end{aligned}
$$

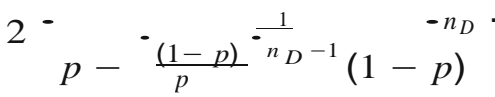

We now look at $\mathrm{W}\left(I\left(n_{D}\right)+1\right)$. Note that $t_{D}\left(q_{D}\right)=I\left(n_{D}\right)+1$ if

$$
q_{D} \in\left(\beta\left(p, I\left(n_{D}\right), n_{D}\right), \beta\left(p, I\left(n_{D}\right)+1, n_{D}\right)\right) .
$$

The total probability of conviction for $t_{D}\left(q_{D}\right)=I\left(n_{D}\right)+1$, if doves Subgroup Deliberate and hawks always convict, is given as follows:

$$
\begin{aligned}
& 1 \quad n D \\
& 2-\left(B\left(p, x, n_{D}\right)+B\left(1-p, x, n_{D}\right)\right) \\
& x=I\left(n_{D}\right)+1 \\
& ={ }_{2}^{1}\left(1-B\left(p, I\left(n_{D}\right), n_{D}\right)-B\left(1-p, I\left(n_{D}\right), n_{D}\right)\right) \text {. }
\end{aligned}
$$

On the other hand, for $q_{D}=\beta\left(p, I\left(n_{D}\right), n_{D}\right)$, the total probability of conviction in the equilibrium $\mathrm{B}$ is given by: 


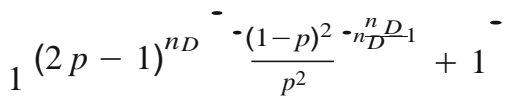

$$
\begin{aligned}
& 2^{-}-^{-}{\frac{(1-p)^{2}}{p^{2}}}^{-\frac{1}{n_{D}^{-1}}}(1-p)^{-n_{D}} \text {. }
\end{aligned}
$$

Now, note that $(44)<(43)$ and $(46) \leq(45)$, for any $p \in\left(\frac{1}{2}, 1\right]$ and $n_{D} \geq 3$. Note that for $n_{D}=1$, the equilibrium $\mathrm{B}$ does not exist so that this case can be ignored. Indeed, $\mathrm{B}$ exists only if $q_{D} \leq \beta(p, 0,1)=1-p$ if $n_{D}=1$. But we have assumed $q D>\frac{1}{2}$.

Step 9 Note the following fact:

Fact 4 : If $W(z+1)-W(z)>0$ then $W(z+2)-W(z+1)>0$, for all $z \in\left\{I\left(n_{D}\right), \ldots, n_{D}-1\right\}$ if $n_{D}$ even, for all $z \in\left\{I\left(n_{D}\right)+1, \ldots, n_{D}-1\right\}$ if $n_{D}$ uneven.

Using the Binomial Formula, for $q_{D}=\beta\left(p, z-1, n_{D}\right)$, we may define and rewrite the following new function, which we use to prove the statement:

$$
\begin{aligned}
& 8\left(p, z, n_{D}\right):={ }_{x=0}^{-n_{D}}\left(B\left(p, x, n_{D}\right)+B\left(1-p, x, n_{D}\right)\right)^{-}\left[y\left(\beta\left(p, z-1, n_{D}\right)\right)\right]^{n_{D}-x}
\end{aligned}
$$

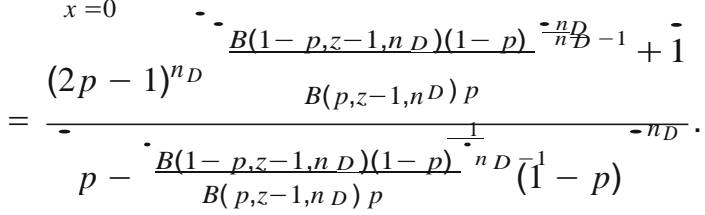

Note that:

$$
\begin{aligned}
\mathrm{W}(z+1)-\mathrm{W}(z)= & 8\left(p, z+1, n_{D}\right)-8\left(p, z, n_{D}\right) \\
& +B\left(p, z-1, n_{D}\right)+B\left(1-p, z-1, n_{D}\right) .
\end{aligned}
$$

Also,

$$
B\left(p, z-1, n_{D}\right)+B\left(1-p, z-1, n_{D}\right)>0, \forall z \in\left\{1, \ldots, n_{D}\right\} .
$$

Note furthermore that

$$
{ }_{2}^{1} 8\left(p, z, n_{D}\right)+{ }_{2}^{1} 8\left(p, z+2, n_{D}\right)>8\left(p, z+1, n_{D}\right) .
$$

Inequality (50) follows from the fact that the function $8\left(p, z, n_{D}\right)$ is decreasing and convex in $z$ over the relevant domain. The latter fact follows from the fact that the following two functions: 
${ }_{988}$ Step 10 From Facts 1,2 and 3 we know that $\mathrm{W}(z)$ is negative at the boundaries. From 989 Fact 4, we know that if $\mathrm{W}(z)$ starts to increase it never decreases again. It follows that 990 it has to be that $\mathrm{W}(z) \leq 0$, for all $z \in\left\{I\left(n_{D}\right), \ldots, n_{D}\right\}$, whether $n_{D}$ is even or uneven.

${ }_{991}$ Step 11 Given that $\mathrm{W}(z) \leq 0$, for all $z \in\left\{I\left(n_{D}\right), \ldots, n_{D}\right\}$, it follows by the argument ${ }_{992}$ given in step 4 that $\partial W\left(q_{j}, q_{D}\right) / \partial q_{j} \leq 0$ for all $q_{D} \in \frac{1}{2}, \beta\left(p, n_{D}-1, n_{D}\right)^{\circ}$, which 993 implies that $W\left(q_{H}, q_{D}\right)>0$ for all $q_{H} \in\left[0, q_{D}\right)$ and $q_{D} \in \frac{1}{2}, \beta\left(p, n_{D}-1, n_{D}\right)$.

995 This complements the part of the proof of Proposition 2 that appears in the main 996 text. We prove in what follows that transiting from $\left(t_{H}-1, t_{D}+1\right)$ to $\left(t_{H}-1, t_{D}\right)$ 997 is beneficial for the preference type $H$ given our assumption that $m>1$. A similar 998 argument shows that transiting from $\left(t_{H}-1, t_{D}+1\right)$ to $\left(t_{H}, t_{D}+1\right)$ is beneficial for 999 the preference type $D$ given our assumption that $m>1$. Assume that

$$
\frac{B\left(p, t_{D}, n_{D}\right)^{-}-\underset{x \geq t_{H-1}}{-n_{H}} B\left(p, x, n_{H}\right)^{-}}{B\left(1-p, t_{D}, n_{D}\right)^{-}-n_{H \geq t_{H-1}} B\left(1-p, x, n_{H}\right)^{-}}<\frac{q_{H}}{1-q_{H}}
$$

1001 and

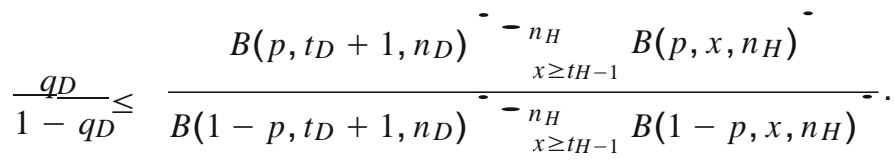


By a standard argument already used in Appendix 2, we furthermore know that by definition, there exists some integer $T \in\{1, \ldots, n\}$ s.t.

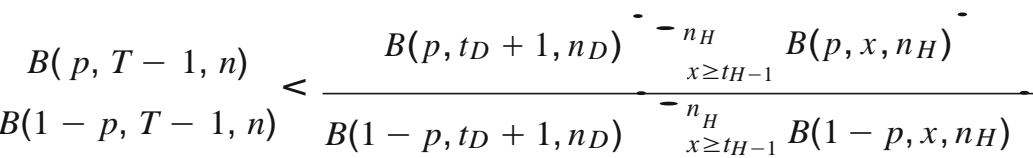

$$
\begin{aligned}
& \leq \frac{B(p, T, n)}{B(1-p, T, n)}
\end{aligned}
$$

and

$$
\begin{aligned}
& \frac{B(p, T-2, n)}{B(1-p, T-2, n)}<\frac{B\left(p, t_{D}, n_{D}\right)^{-}-n_{H \geq t_{H-1}} B\left(p, x, n_{H}\right)^{-}}{B\left(1-p, t_{D}, n_{D}\right)^{-n_{H}} \begin{array}{c}
x \geq t_{H-1} \\
x\left(1-p, x, n_{H}\right)
\end{array}} \\
& \leq \frac{B(p, T-1, n)}{B(1-p, T-1, n)} \text {. }
\end{aligned}
$$

Now, the inequalities (54), (55), (56) and (57) imply that there is some integer $T \in\{1, \ldots, n\}$ s.t.

$$
\frac{B(p, T-2, n)}{B(1-p, T-2, n)}<\frac{q_{H}}{1-q_{H}}<\frac{q_{D}}{1-q_{D}} \leq \frac{B(p, T, n)}{B(1-p, T, n)},
$$

which contradicts our assumption that $m>1$. It follows that (54) and (55) cannot be true.

\section{Proposition 3: reactive SSDEs vs reactive SPDE}

Step 1 The unique reactive SPDE is characterized by a dove threshold $T^{n}{ }^{n}$. Now, there

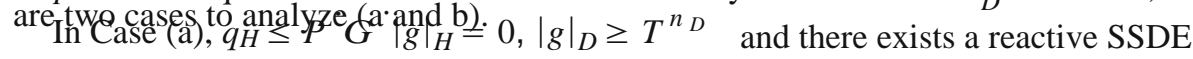
given by $t_{H}=0$ and $t_{D}=T_{D}^{n_{D}}$. This latter reactive SSDE is outcome equivalent to the unique reactive simple SPDE. If there exists any other reactive SSDE, then by Proposition 2, it is strongly Pareto dominant w.r.t. the reactive SSDE in which $t_{H}=0$ and $t_{B} \overline{\overline{\mathrm{In}}} T_{D}^{n_{D}}$, and thus also strongly Pareto dominant $w_{\text {. }}$.t. the unique reactive SPDE. Step 2 In Case (b), $q_{H}>\left.P G_{g}\right|_{H}=0,|g|_{D} \geq T$ and there thus exists no

reactive SSDE given by $t_{H}=0$ and $t_{D}=T_{D}^{n_{D}}$. We know however from Lemma 6 that there exists some reactive SSDE. We now conduct an argument based on a hypothetical adjustment process. Start from the reactive SSD profile in which $t_{H}=0$ and $t_{D}=T_{D}^{n_{D}}$. We know that this profile (although it is not an equilibrium profile) yields a payoff to each preference type that is equivalent to that received in the unique reactive SPDE. Now, let hawks choose their collective best response to $T_{D}^{n_{D}}$, i.e. $t_{H}^{B R}\left(T_{D}^{n_{D}}\right)$. We know that the latter is strictly larger than 0 given that $q_{H}>P \cdot \dot{G} \cdot|g|_{H}=0,|g|_{D} \geq_{D} T^{\dot{n}_{D}}$.

This adjustment is strictly beneficial to hawks and also to doves, given that hawks become more lenient. In a further step, let doves revise their threshold and choose their 
own best response $t^{B R}\left(t^{B R}\left(T_{D}^{n_{D}}\right)\right) \cdot \mathrm{HA}^{\mathrm{A}} \mathrm{gai}$, the adjustment is by definition beneficial to doves as well as to hawks, as doves become weakly harsher. Repeat the adjustment of the hawks, etc.

This process of mutual adjustment converges to a reactive SSDE, and every step of the adjustment process is strictly welfare improving for both preference types. It follows that the reactive SSDE to which our adjustment process converges is strongly Pareto dominant w.r.t. the unique reactive SPDE. Note furthermore than any other reactive SSDE is less polarized than this first reactive SSDE and thus, by Proposition 2, strongly Pareto improving w.r.t. the latter. It follows that any reactive SSDE is strongly Pareto dominant w.r.t. the unique reactive SPDE.

\section{References}

Austen-Smith D, Feddersen TJ (2006) Deliberation, preference uncertainty and voting rules. Am Polit Sci Rev 100:209-217

Coughlan PJ (2000) In defense of unanimous jury verdicts : mistrials, communication and strategic voting. Am Polit Sci Rev 94:375-393

Deimen I, Ketelaar, F, Le Quement MT (2014) Consistency and communication in committees, mimeo Doraszelski U, Gerardi D, Squintani F (2006) Communication and voting with doubled sided information, Contrib Theor Econom, 3

Duggan J, Martinelli C (2001) A Bayesian model of voting in juries. Games Econ Behav 37:259-294

Feddersen TJ, Pesendorfer W (1998) Convicting the innocent: the inferiority of unanimous jury verdicts under strategic voting. Am Polit Sci Rev 92:23-35

Gerardi D (2000) Jury verdicts and preference diversity. Am Polit Sci Rev 94:395-406

Gerardi D, Yariv L (2007) Deliberative voting. J Econ Theor 134:317-338

Hummel P (2010) Deliberation in large juries with diverse preferences. Public Choice 150:595-608

Le Quement MT (2012) Communication compatible voting rules. Theor Decis 74:479-507

Meirowitz A (2002) Informative voting and Condorcet jury theorems with a continuum of types. Soc Choice Welfare 19:219-236

Meirowitz A (2007) In defense of exclusionary deliberation: communication and voting with private beliefs and values. J Theor Polit 19:301-327

Van Weelden R (2008) Deliberation rules and voting. Q J Polit Sci 3:83-88

Wolinsky A (2002) Eliciting information from multiple experts. Games Econ Behav 41:1419-1431 